\title{
A Type II Supernova Hubble Diagram from the CSP-I, SDSS-II, and SNLS Surveys
}

\author{
T. de Jaeger ${ }^{1,2,3}$, S. González-Gaitán ${ }^{1,4}$, M. Hamuy ${ }^{1,2}$, L. Galbany ${ }^{5}$, J. P. Anderson ${ }^{6}$, M. M. Phillips ${ }^{7}$, M. D. Stritzinger ${ }^{8}$, \\ R. G. Carlberg 9 , M. Sullivan ${ }^{10}$, C. P. Gutiérrez ${ }^{1,2,6}$, I. M. Hook ${ }^{11}$, D. Andrew Howell ${ }^{12,13}$, E. Y. Hsiao ${ }^{7,8,14}$, H. Kuncarayakti ${ }^{1,2}$, \\ V. Ruhlmann-Kleider ${ }^{15}$, G. Folatelli ${ }^{16}$, C. Pritchet ${ }^{17}$, and S. Basa ${ }^{18}$ \\ ${ }^{1}$ Millennium Institute of Astrophysics, Santiago, Chile; dthomas@das.uchile.cl, tdejaeger@berkeley.edu \\ ${ }^{2}$ Departamento de Astronomía, Universidad de Chile, Camino el Observatorio 1515, Santiago, Chile \\ ${ }^{3}$ Department of Astronomy, University of California, Berkeley, CA 94720-3411, USA \\ ${ }^{4}$ Center for Mathematical Modelling, University of Chile, Beaucheff 851, Santiago, Chile \\ ${ }^{5}$ PITT PACC, Department of Physics and Astronomy, University of Pittsburgh, Pittsburgh, PA 15260, USA \\ ${ }^{6}$ European Southern Observatory, Alonso de Córdova 3107, Casilla 19, Santiago, Chile \\ ${ }^{7}$ Las Campanas Observatory, Carnegie Observatories, Casilla 601, La Serena, Chile \\ ${ }^{8}$ Department of Physics and Astronomy, Aarhus University, Ny Munkegade 120, DK-8000 Aarhus C, Denmark \\ ${ }^{9}$ Department of Astronomy and Astrophysics, University of Toronto, 50 St. George Street, Toronto, ON, M5S 3H4, Canada \\ ${ }^{10}$ Department of Physics and Astronomy, University of Southampton, Southampton, SO17 1BJ, UK \\ ${ }_{11}$ Physics Department, Lancaster University, Lancaster LA1 4 YB, UK \\ ${ }^{12}$ Las Cumbres Observatory Global Telescope Network, 6740 Cortona Drive, Suite 102, Goleta, CA 93117, USA \\ ${ }^{13}$ Department of Physics, University of California, Santa Barbara, Broida Hall, Mail Code 9530, Santa Barbara, CA 93106-9530, USA \\ ${ }^{14}$ Department of Physics, Florida State University, Tallahassee, FL 32306, USA \\ ${ }^{15}$ CEA, Centre de Saclay, irfu SPP, F-91191 Gif-sur-Yvette, France \\ ${ }^{16}$ Instituto de Astrofísica de La Plata, CONICET, Paseo del Bosque S/N, B1900FWA, La Plata, Argentina \\ ${ }_{17}^{17}$ University of Victoria, Department of Physics and Astronomy, P.O. Box 1700, Stn CSC, Victoria, BC, V8W 2Y2, Canada \\ 18 Aix Marseille Université, CNRS, LAM (Laboratoire d'Astrophysique de Marseille) UMR 7326, F-13388, Marseille, France \\ Received 2016 July 13; revised 2016 November 20; accepted 2016 December 15; published 2017 January 27
}

\begin{abstract}
The coming era of large photometric wide-field surveys will increase the detection rate of supernovae by orders of magnitude. Such numbers will restrict spectroscopic follow-up in the vast majority of cases, and hence new methods based solely on photometric data must be developed. Here, we construct a complete Hubble diagram of Type II supernovae (SNe II) combining data from three different samples: the Carnegie Supernova Project-I, the Sloan Digital Sky Survey II SN, and the Supernova Legacy Survey. Applying the Photometric Color Method (PCM) to 73 SNe II with a redshift range of 0.01-0.5 and with no spectral information, we derive an intrinsic dispersion of 0.35 mag. A comparison with the Standard Candle Method (SCM) using 61 SNe II is also performed and an intrinsic dispersion in the Hubble diagram of 0.27 mag, i.e., $13 \%$ in distance uncertainties, is derived. Due to the lack of good statistics at higher redshifts for both methods, only weak constraints on the cosmological parameters are obtained. However, assuming a flat universe and using the PCM, we derive the universe's matter density: $\Omega_{m}=0.32_{-0.21}^{+0.30}$ providing a new independent evidence for dark energy at the level of two sigma.
\end{abstract}

Key words: distance scale - galaxies: distances and redshifts - supernovae: general

\section{Introduction}

One of the most important investigations in astronomy is to understand the formation and the composition of our universe. Achieving this goal is very challenging but can be done by measuring distances using astrophysical sources for which the absolute magnitude is known (a.k.a. standard candles), and using the Hubble diagram as a classical cosmological test.

For more than two decades, Type Ia supernovae (hereafter SNe Ia; Minkowski 1941; Filippenko 1997; Howell 2011 and references therein) have been used as standard candles in cosmology (e.g., Phillips 1993; Hamuy et al. 1996; Riess et al. 1996; Perlmutter et al. 1997), and led to the revolutionary discovery of the accelerated expansion of the universe driven by an unknown force attributed to dark energy (Riess et al. 1998; Schmidt et al. 1998; Perlmutter et al. 1999).

* This paper includes data gathered with the $6.5 \mathrm{~m}$ Magellan Telescopes, with the du Pont and Swope telescopes located at Las Campanas Observatory, Chile; and the Gemini Observatory, Cerro Pachon, Chile (Gemini Program N-2005AQ-11, GN-2005B-Q-7, GN-2006A-Q-7, GS-2005A-Q-11, GS-2005B-Q-6, and GS-2008B-Q-56). Based on observations collected at the European Organization for Astronomical Research in the Southern Hemisphere, Chile (ESO Programmes 076.A-0156,078.D-0048, 080.A-0516, and 082.A-0526).
SNe Ia cosmology today has reached a mature state in which systematic errors dominate the overall error budget of the cosmological parameters (e.g., Conley et al. 2011; Rubin et al. 2013; Betoule et al. 2014) and further improvement to constrain the nature of the dark energy requires developing as many independent methods as possible.

One of the most interesting independent techniques to derive accurate distances and measure cosmological parameters is the use of Type II supernovae (hereafter SNe II). ${ }^{19}$ Even if both SNe Ia and SNe II cosmology in general use the same surveys and share some systematic uncertainties like the photometric calibration, other systematic errors are different such as the redshift evolution uncertainties. Furthermore, SNe II are the result of the same physical mechanism, and their progenitors are better understood than those of SNe Ia (Smartt et al. 2009).

To date, several methods have been developed to standardize SNe II, such as

\footnotetext{
19 Throughout the rest of the text we refer to SNe II as the two historical groups, SNe IIP and SNe IIL, since recent studies showed that the SNe II family forms a continuous class (Anderson et al. 2014; Sanders et al. 2015; Valenti et al. 2016). Note that Arcavi et al. (2012) and Faran et al. (2014a, 2014b) have argued for two separate populations.
} 
1. the "Expanding Photosphere Method" (EPM) developed by Kirshner \& Kwan (1974),

2. the "Spectral-fitting Expanding Atmosphere Method" (SEAM; Baron et al. 2004 and updated in Dessart et al. 2008),

3. the "Standard Candle Method" (SCM) introduced by Hamuy \& Pinto (2002),

4. the "Photospheric Magnitude Method" (PMM), which is a generalization of the SCM over various epochs (Rodríguez et al. 2014), and

5. the most recent technique, the "Photometric Color Method" (PCM; de Jaeger et al. 2015).

In this paper, we focus our effort on two different methods: the SCM, which is the most common method used to derive SN II distances and thus makes the comparison with other works easier, and the PCM, which is the only purely photometric method in the literature, i.e., it does not require observed spectra. The EPM and the SEAM methods are not discussed in this paper because they require corrections factors computed from model atmospheres (Eastman et al. 1996; Dessart \& Hillier 2005).

The SCM is a powerful method based on both photometric and spectroscopic input parameters, and enables a decrease in the scatter in the Hubble diagram from $\sim 1$ mag to levels of 0.3 mag (Hamuy \& Pinto 2002) and to derive distances with a precision of $\sim 14 \%$. This method is mainly built on the correlation between the SN II luminosity and the photospheric expansion velocity 50 days post-explosion. More luminous SNe II have their hydrogen recombination front at a larger radius and thus the velocity of the photosphere will be greater in a homologous expansion (Kasen \& Woosley 2009). Many other works have used an updated version of the SCM where a color correction is added in order to take into account the hostgalaxy extinction. All these studies (Nugent et al. 2006; Poznanski et al. 2009, 2010; D'Andrea et al. 2010; Olivares et al. 2010; de Jaeger et al. 2015) have confirmed the use of SNe II as distance indicators, finding similar dispersion in the Hubble diagram (0.25-0.30 mag).

Recently, de Jaeger et al. (2015) suggested a new method using corrected magnitudes derived only from photometry. In this method, instead of using the photospheric expansion velocity, the standardization is done using the second, shallower slope in the light curve after maximum, $s_{2}$, which corresponds to the plateau for the SNe IIP (Anderson et al. 2014). Anderson et al. (2014) found that more luminous SNe II have higher $s_{2}$ (steeper decline, $>1.15$ mag per 100 days), confirming previous studies finding that traditional SNe IIL are more luminous than SNe IIP (Patat et al. 1994; Richardson et al. 2014). Using this correlation and adding a color term, de Jaeger et al. (2015) succeeded in reducing the scatter in the low-redshift SNe II Hubble diagram ( $z=0.01-0.04)$ to a level of $\sim 0.4 \mathrm{mag}$ ( $\pm 0.05 \mathrm{mag}$ ), which corresponds to a precision of $18 \%$ in distances.

Better comprehension of our universe requires the observation of more distant SNe II. Differences between the expansion histories are extremely small and distinguishing between them will require measurements extending far back in time. The main purpose of the current work is to build a Hubble diagram using the SCM and the PCM as achieved in de Jaeger et al. (2015) but adding higher redshift SN samples such as the Sloan Digital Sky Survey II Supernova Survey (SDSS-II SN; Frieman et al. 2008; Sako et al. 2014) and the Supernova Legacy Survey (SNLS; Astier et al. 2006; Perrett et al. 2010).

The paper is organized as follows. In Section 2 we give a description of the data set and Section 3 describes our procedure for performing $\mathrm{K}$-corrections and S-corrections, together with line-of-sight extinction corrections from our own Milky Way. Section 4 presents the Hubble diagram obtained using the PCM while in Section 5 we use the SCM. In Section 6 we discuss our results and conclude with a summary in Section 7.

\section{Data Sample}

In this paper, we use data from three different projects: the Carnegie Supernova Project-I ${ }^{20}$ (CSP-I; Hamuy et al. 2006), the SDSS-II SN Survey ${ }^{21}$ (Frieman et al. 2008), and the Supernova Legacy Survey ${ }^{22}$ (Astier et al. 2006; Perrett et al. 2010). These three surveys all used very similar Sloan optical filters, permitting a minimization of the systematic errors. Our sample is listed in Table 1.

\subsection{Carnegie Supernova Project-I}

The CSP-I had guaranteed access to $\sim 300$ nights per year between 2004 and 2009 on the Swope $1 \mathrm{~m}$ and du Pont $2.5 \mathrm{~m}$ telescopes at the Las Campanas Observatory (LCO), both equipped with high-performance $\mathrm{CCD}$ and IR cameras and CCD spectrographs. This observation time allowed the CSP-I to obtain optical-band light curves for $67 \mathrm{SNe}$ II (with $z \leqslant 0.04$ ) with good temporal coverage and more than 500 visual-wavelength spectra for these same objects.

The optical photometry $(u, g, r, i)$ was obtained after data processing via standard reduction techniques. The final magnitudes were derived relative to local sequence stars and calibrated from observations of standard stars (Smith et al. 2002) and are expressed in the natural photometric system of the Swope+CSP-I bands. The spectra were also reduced and calibrated in a standard manner using IRAF. ${ }^{23} \mathrm{~A}$ full description can be found in Hamuy et al. (2006), Contreras et al. (2010), Stritzinger et al. (2011), and Folatelli et al. (2013).

From the CSP-I sample, we remove six outliers. Three were described in de Jaeger et al. (2015) but SN 2005hd has no clear explosion date defined, SN 2008bp is identified as an outlier by Anderson et al. (2014), and SN 2009au was classified at the beginning as an $\mathrm{SNe}$ IIn showing strong interaction. Thus, the total sample used is composed of $61 \mathrm{SNe}$ II.

\subsection{Sloan Digital Sky Survey-II SN Survey}

The SDSS-II SN Survey operated for three years, from 2005 September to 2007 November. Using the $2.5 \mathrm{~m}$ telescope at the Apache Point Observatory in New Mexico (Gunn et al. 2006), the survey repeatedly imaged the same region of the sky around southern equatorial stripe 82 (Stoughton et al. 2002). This survey observed about 80 spectroscopically confirmed corecollapse $\mathrm{SNe}$ but the main driver of this project was the study of SNe Ia, involving the acquisition of only one or two spectra per SNe II.

\footnotetext{
${ }^{20}$ http://csp.obs.carnegiescience.edu/

21 http://classic.sdss.org/supernova/aboutsupernova.html

22 http://cfht.hawaii.edu/SNLS/

23 IRAF is distributed by the National Optical Astronomy Observatory, which is operated by the Association of Universities for Research in Astronomy (AURA) under cooperative agreement with the National Science Foundation.
} 
Table 1

Supernova Sample

\begin{tabular}{|c|c|c|c|c|c|c|c|c|c|c|}
\hline $\mathrm{SN}$ & $\begin{array}{c}\mathrm{AvG} \\
\mathrm{mag}\end{array}$ & $z_{\text {helio }}$ & $z_{\mathrm{CMB}}(\mathrm{err})$ & $\begin{array}{c}\text { Explosion Date } \\
\text { MJD }\end{array}$ & $\begin{array}{c}s_{2} \\
\text { mag } 100 \text { days }^{-1}\end{array}$ & $\begin{array}{c}v_{\mathrm{H} \beta^{\mathrm{a}}} \\
\mathrm{km} \mathrm{s}^{-1}\end{array}$ & $\begin{array}{c}\mu_{\mathrm{PCM}} \\
\mathrm{mag}\end{array}$ & $\begin{array}{c}\mu_{\mathrm{SCM}} \\
\mathrm{mag}\end{array}$ & Campaign $^{\mathrm{b}}$ & Methods \\
\hline $2004 \mathrm{er}^{\mathrm{c}}$ & 0.070 & 0.0147 & $0.0139(0.00011)$ & $53271.8(4.0)$ & $0.41(0.03)$ & $8100(170)$ & $33.37(0.06)$ & $33.84(0.06)$ & CSP-I & $\overline{\mathrm{PCM} / \mathrm{SCM}}$ \\
\hline $2004 \mathrm{fb}$ & 0.173 & 0.0203 & $0.0197(0.00009)$ & $53242.6(4.0)$ & $0.47(0.10)$ & $\cdots$ & $\cdots$ & $\cdots$ & CSP-I & $\mathrm{PCM} / \mathrm{SCM}$ \\
\hline $2004 f^{c}{ }^{c}$ & 0.069 & 0.0061 & $0.0052(0.00002)$ & $53293.5(10.0)$ & $-0.08(0.03)$ & $\ldots$ & $\ldots$ & $\ldots$ & CSP-I & $\mathrm{PCM} / \mathrm{SCM}$ \\
\hline $2004 f x^{c}$ & 0.282 & 0.0089 & $0.0089(0.00001)$ & $53303.5(4.0)$ & $0.70(0.05)$ & $\ldots$ & $\ldots$ & $\ldots$ & CSP-I & $\mathrm{PCM} / \mathrm{SCM}$ \\
\hline $2005 J^{\mathrm{c}}$ & 0.075 & 0.0139 & $0.0151(0.00001)$ & $53382.7(7.0)$ & $0.59(0.01)$ & $6160(160)$ & $33.84(0.06)$ & $33.98(0.07)$ & CSP-I & $\mathrm{PCM} / \mathrm{SCM}$ \\
\hline $2005 K$ & 0.108 & 0.0273 & $0.0284(0.00010)$ & $53369.8(7.0)$ & $1.06(0.08)$ & $5490(260)$ & $35.95(0.07)$ & $35.64(0.10)$ & CSP-I & $\mathrm{PCM} / \mathrm{SCM}$ \\
\hline $2005 Z^{c}$ & 0.076 & 0.0192 & $0.0203(0.00003)$ & $53396.7(8.0)$ & $1.30(0.02)$ & $7510(160)$ & $34.42(0.06)$ & $34.62(0.07)$ & CSP-I & $\mathrm{PCM} / \mathrm{SCM}$ \\
\hline 2005af & 0.484 & 0.0019 & $0.0027(0.00001)$ & $53323.8(15.0)$ & $-0.03(0.13)$ & $\ldots$ & $\cdots$ & $\cdots$ & CSP-I & $\mathrm{PCM} / \mathrm{SCM}$ \\
\hline $2005 \mathrm{an}^{\mathrm{c}}$ & 0.262 & 0.0107 & $0.0118(0.00010)$ & $53426.7(4.0)$ & $1.82(0.05)$ & $6650(170)$ & $34.02(0.07)$ & $33.88(0.07)$ & CSP-I & $\mathrm{PCM} / \mathrm{SCM}$ \\
\hline $2005 \mathrm{dk}^{\mathrm{c}}$ & 0.134 & 0.0157 & $0.0154(0.00008)$ & $53599.5(6.0)$ & $0.86(0.03)$ & $6900(170)$ & $33.74(0.06)$ & $33.94(0.07)$ & CSP-I & $\mathrm{PCM} / \mathrm{SCM}$ \\
\hline $2005 \mathrm{dn}^{c}$ & 0.140 & 0.0094 & $0.0090(0.00006)$ & $53601.5(6.0)$ & $1.22(0.04)$ & $\cdots$ & $\cdots$ & $\cdots$ & CSP-I & $\mathrm{PCM} / \mathrm{SCM}$ \\
\hline $2005 \mathrm{dt}$ & 0.079 & 0.0256 & $0.0570(0.00008)$ & $53605.6(9.0)$ & $-0.01(0.07)$ & $4500(190)$ & $35.20(0.06)$ & $35.03(0.08)$ & CSP-I & $\mathrm{PCM} / \mathrm{SCM}$ \\
\hline $2005 \mathrm{dw}^{\mathrm{c}}$ & 0.062 & 0.0176 & $0.0166(0.00007)$ & $53603.6(9.0)$ & $0.76(0.03)$ & $5040(310)$ & $34.70(0.07)$ & $34.41(0.11)$ & CSP-I & $\mathrm{PCM} / \mathrm{SCM}$ \\
\hline $2005 d x^{c}$ & 0.066 & 0.0267 & $0.0264(0.00010)$ & $53615.9(7.0)$ & $0.58(0.04)$ & $4660(190)$ & $35.80(0.07)$ & $35.47(0.08)$ & CSP-I & $\mathrm{PCM} / \mathrm{SCM}$ \\
\hline $2005 \mathrm{dz}^{\mathrm{c}}$ & 0.223 & 0.0190 & $0.0177(0.00009)$ & $53619.5(4.0)$ & $0.33(0.02)$ & $5965(170)$ & $34.69(0.06)$ & $34.73(0.07)$ & CSP-I & $\mathrm{PCM} / \mathrm{SCM}$ \\
\hline $2005 \mathrm{es}^{\mathrm{c}}$ & 0.228 & 0.0376 & $0.0364(0.00018)$ & $53638.7(10.0)$ & $\ldots$ & $5055(190)$ & $\ldots$ & $35.64(0.08)$ & CSP-I & $\mathrm{PCM} / \mathrm{SCM}$ \\
\hline $2005 \mathrm{gk}^{\mathrm{c}}$ & 0.154 & 0.0292 & $0.0286(0.00010)$ & $53647.8(1.0)$ & $0.62(0.06)$ & $\cdots$ & $35.41(0.06)$ & $\ldots$ & CSP-I & $\mathrm{PCM} / \mathrm{SCM}$ \\
\hline $20051 w^{c}$ & 0.135 & 0.0257 & $0.0269(0.00013)$ & $53716.8(5.0)$ & $1.27(0.05)$ & $7375(180)$ & $34.88(0.07)$ & $35.01(0.07)$ & CSP-I & $\mathrm{PCM} / \mathrm{SCM}$ \\
\hline $2005 \mathrm{me}$ & 0.070 & 0.0224 & $0.0218(0.00005)$ & $53721.6(6.0)$ & $0.99(0.10)$ & $5700(240)$ & $35.41(0.07)$ & $35.23(0.08)$ & CSP-I & $\mathrm{PCM} / \mathrm{SCM}$ \\
\hline $2006 Y^{c}$ & 0.354 & 0.0336 & $0.0341(0.00010)$ & $53766.5(4.0)$ & $0.90(0.14)$ & $6890(160)$ & $35.73(0.08)$ & $35.92(0.07)$ & CSP-I & $\mathrm{PCM} / \mathrm{SCM}$ \\
\hline $2006 \mathrm{ai}^{\mathrm{c}}$ & 0.347 & 0.0152 & $0.0154(0.00010)$ & $53781.8(5.0)$ & $1.15(0.05)$ & $6430(160)$ & $33.89(0.06)$ & $33.87(0.06)$ & CSP-I & $\mathrm{PCM} / \mathrm{SCM}$ \\
\hline $2006 \mathrm{bc}^{\mathrm{c}}$ & 0.562 & 0.0045 & $0.0049(0.00004)$ & $53815.5(4.0)$ & $\ldots$ & $\ldots$ & $\ldots$ & $\ldots$ & CSP-I & $\mathrm{PCM} / \mathrm{SCM}$ \\
\hline $2006 \mathrm{be}^{\mathrm{c}}$ & 0.080 & 0.0071 & $0.0075(0.00003)$ & $53805.8(6.0)$ & $0.11(0.03)$ & $\ldots$ & $\ldots$ & $\ldots$ & CSP-I & $\mathrm{PCM} / \mathrm{SCM}$ \\
\hline $2006 \mathrm{bl}^{\mathrm{c}}$ & 0.144 & 0.0324 & $0.0328(0.00016)$ & $53823.8(6.0)$ & $1.13(0.09)$ & $6550(180)$ & $35.06(0.07)$ & $35.30(0.07)$ & CSP-I & $\mathrm{PCM} / \mathrm{SCM}$ \\
\hline $2006 e^{c}$ & 0.167 & 0.0154 & $0.0145(0.00009)$ & $53961.8(4.0)$ & $-0.61(0.06)$ & $3330(170)$ & $33.96(0.06)$ & $33.54(0.09)$ & CSP-I & $\mathrm{PCM} / \mathrm{SCM}$ \\
\hline $2006 \mathrm{it}^{\mathrm{c}}$ & 0.273 & 0.0155 & $0.0145(0.00007)$ & $54006.5(3.0)$ & $\cdots$ & $\cdots$ & $\cdots$ & $\cdots$ & CSP-I & $\mathrm{PCM} / \mathrm{SCM}$ \\
\hline $2006 \mathrm{~ms}^{\mathrm{c}}$ & 0.095 & 0.0151 & $0.0147(0.00007)$ & $54034.0(12.0)$ & $-0.53(0.06)$ & $\ldots$ & $34.23(0.06)$ & $\ldots$ & CSP-I & $\mathrm{PCM} / \mathrm{SCM}$ \\
\hline $2006 \mathrm{qr}^{\mathrm{c}}$ & 0.126 & 0.0145 & $0.0155(0.00007)$ & $54062.8(7.0)$ & $0.44(0.02)$ & $5150(160)$ & $34.82(0.05)$ & $34.67(0.07)$ & CSP-I & $\mathrm{PCM} / \mathrm{SCM}$ \\
\hline $2007 \mathrm{P}^{\mathrm{c}}$ & 0.111 & 0.0407 & $0.0419(0.00010)$ & $54118.7(3.0)$ & $0.28(0.12)$ & $5710(220)$ & $35.84(0.08)$ & $35.87(0.08)$ & CSP-I & $\mathrm{PCM} / \mathrm{SCM}$ \\
\hline $2007 \mathrm{U}^{\mathrm{c}}$ & 0.145 & 0.0260 & $0.0260(0.00003)$ & $54134.6(6.0)$ & $1.40(0.04)$ & $7050(170)$ & $34.91(0.06)$ & $34.96(0.09)$ & CSP-I & $\mathrm{PCM} / \mathrm{SCM}$ \\
\hline $2007 \mathrm{~W}^{\mathrm{c}}$ & 0.141 & 0.0097 & $0.0107(0.00007)$ & $54136.8(7.0)$ & $-0.69(0.06)$ & $3270(160)$ & $33.78(0.06)$ & $33.79(0.06)$ & CSP-I & $\mathrm{PCM} / \mathrm{SCM}$ \\
\hline $2007 \mathrm{aa}^{\mathrm{c}}$ & 0.072 & 0.0049 & $0.0061(0.00008)$ & $54135.8(5.0)$ & $-0.09(0.09)$ & $\ldots$ & $\ldots$ & $\ldots$ & CSP-I & $\mathrm{PCM} / \mathrm{SCM}$ \\
\hline $2007 \mathrm{ab}^{\mathrm{c}}$ & 0.730 & 0.0235 & $0.0236(0.00004)$ & $54123.8(6.0)$ & $2.87(0.05)$ & $8580(190)$ & $35.30(0.06)$ & $35.08(0.07)$ & CSP-I & $\mathrm{PCM} / \mathrm{SCM}$ \\
\hline $2007 a v^{c}$ & 0.099 & 0.0046 & $0.0058(0.00008)$ & $54175.7(5.0)$ & $0.22(0.04)$ & $\ldots$ & $\ldots$ & $\ldots$ & CSP-I & $\mathrm{PCM} / \mathrm{SCM}$ \\
\hline $2007 \mathrm{hm}^{\mathrm{c}}$ & 0.172 & 0.0251 & $0.0241(0.00008)$ & $54335.6(6.0)$ & $1.30(0.04)$ & $6260(200)$ & $35.92(0.06)$ & $35.74(0.07)$ & CSP-I & $\mathrm{PCM} / \mathrm{SCM}$ \\
\hline $2007 \mathrm{il}^{\mathrm{c}}$ & 0.129 & 0.0215 & $0.0205(0.00008)$ & $54349.8(4.0)$ & $-0.42(0.03)$ & $6110(160)$ & $34.81(0.06)$ & $34.66(0.08)$ & CSP-I & $\mathrm{PCM} / \mathrm{SCM}$ \\
\hline 2007 it & 0.316 & 0.0040 & $0.0047(0.00050)$ & $54348.5(1.0)$ & $\ldots$ & $\ldots$ & $\ldots$ & $\ldots$ & CSP-I & $\mathrm{PCM} / \mathrm{SCM}$ \\
\hline $2007 \mathrm{oc}^{\mathrm{c}}$ & 0.061 & 0.0048 & $0.0039(0.00007)$ & $54388.5(3.0)$ & $1.31(0.03)$ & $\ldots$ & $\ldots$ & $\ldots$ & CSP-I & $\mathrm{PCM} / \mathrm{SCM}$ \\
\hline $2007 \mathrm{od}^{\mathrm{c}}$ & 0.100 & 0.0058 & $0.0045(0.00006)$ & $54402.6(5.0)$ & $0.70(0.06)$ & $\ldots$ & $\ldots$ & $\ldots$ & CSP-I & $\mathrm{PCM} / \mathrm{SCM}$ \\
\hline $2007 \mathrm{sq}^{\mathrm{c}}$ & 0.567 & 0.0153 & $0.0162(0.00007)$ & $54421.8(3.0)$ & $0.34(0.05)$ & $7500(170)$ & $34.28(0.06)$ & $34.69(0.06)$ & CSP-I & $\mathrm{PCM} / \mathrm{SCM}$ \\
\hline $2008 \mathrm{~F}^{\mathrm{c}}$ & 0.135 & 0.0183 & $0.0177(0.00008)$ & $54470.6(6.0)$ & $-0.63(0.08)$ & $4825(170)$ & $34.77(0.07)$ & $34.88(0.08)$ & CSP-I & $\mathrm{PCM} / \mathrm{SCM}$ \\
\hline $2008 \mathrm{M}^{\mathrm{c}}$ & 0.124 & 0.0076 & $0.0079(0.00002)$ & $54471.7(9.0)$ & $0.23(0.05)$ & $\ldots$ & $\ldots$ & $\ldots$ & CSP-I & $\mathrm{PCM} / \mathrm{SCM}$ \\
\hline $2008 W^{c}$ & 0.267 & 0.0192 & $0.0201(0.00016)$ & $54485.8(6.0)$ & $0.26(0.04)$ & $5760(160)$ & $34.92(0.06)$ & $34.65(0.07)$ & CSP-I & $\mathrm{PCM} / \mathrm{SCM}$ \\
\hline $2008 \mathrm{ag}^{\mathrm{c}}$ & 0.229 & 0.0148 & $0.0147(0.00002)$ & $54479.8(6.0)$ & $-0.32(0.03)$ & $4760(160)$ & $33.42(0.06)$ & $33.42(0.07)$ & CSP-I & $\mathrm{PCM} / \mathrm{SCM}$ \\
\hline $2008 \mathrm{aw}^{\mathrm{c}}$ & 0.111 & 0.0104 & $0.0114(0.00007)$ & $54517.8(10.0)$ & $1.49(0.05)$ & $6900(160)$ & $33.22(0.06)$ & $33.19(0.06)$ & CSP-I & $\mathrm{PCM} / \mathrm{SCM}$ \\
\hline
\end{tabular}


Table 1

(Continued)

\begin{tabular}{|c|c|c|c|c|c|c|c|c|c|c|}
\hline $\mathrm{SN}$ & $\begin{array}{c}\mathrm{AvG} \\
\mathrm{mag}\end{array}$ & $z_{\text {helio }}$ & $z_{\mathrm{CMB}}(\mathrm{err})$ & $\begin{array}{c}\text { Explosion Date } \\
\text { MJD }\end{array}$ & $\begin{array}{c}s_{2} \\
\text { mag } 100 \text { days }^{-1}\end{array}$ & $\begin{array}{c}v_{\mathrm{H} \beta^{a}}{ }^{1} \\
\mathrm{~km} \mathrm{~s}{ }^{-1}\end{array}$ & $\begin{array}{c}\mu_{\mathrm{PCM}} \\
\mathrm{mag}\end{array}$ & $\begin{array}{c}\mu_{\mathrm{SCM}} \\
\mathrm{mag}\end{array}$ & Campaign $^{\mathrm{b}}$ & Methods \\
\hline $2008 \mathrm{bh}^{\mathrm{c}}$ & 0.060 & 0.0145 & $0.0154(0.00007)$ & $54543.5(5.0)$ & $0.65(0.04)$ & $6470(180)$ & $34.55(0.06)$ & $34.67(0.07)$ & CSP-I & $\overline{\mathrm{PCM} / \mathrm{SCM}}$ \\
\hline $2008 \mathrm{bk}^{\mathrm{c}}$ & 0.054 & 0.0008 & $-0.0001(0.00006)$ & $54542.9(6.0)$ & $\ldots$ & $\ldots$ & $\ldots$ & $\ldots$ & CSP-I & $\mathrm{PCM} / \mathrm{SCM}$ \\
\hline $2008 \mathrm{br}$ & 0.255 & 0.0101 & $0.0111(0.00007)$ & $54555.7(9.0)$ & $-0.65(0.05)$ & $2420(180)$ & $34.19(0.06)$ & $33.33(0.12)$ & CSP-I & $\mathrm{PCM} / \mathrm{SCM}$ \\
\hline $2008 \mathrm{bu}^{\mathrm{c}}$ & 1.149 & 0.0221 & $0.0222(0.00003)$ & $54566.8(5.0)$ & $-0.07(0.33)$ & $5930(200)$ & $34.59(0.14)$ & $34.96(0.09)$ & CSP-I & $\mathrm{PCM} / \mathrm{SCM}$ \\
\hline $2008 \mathrm{ga}^{\mathrm{c}}$ & 1.865 & 0.0155 & $0.0153(0.00001)$ & $54711.8(4.0)$ & $1.01(0.05)$ & $5550(270)$ & $34.19(0.07)$ & $34.03(0.09)$ & CSP-I & $\mathrm{PCM} / \mathrm{SCM}$ \\
\hline $2008 \mathrm{gi}^{\mathrm{c}}$ & 0.181 & 0.0244 & $0.0237(0.00012)$ & $54742.7(9.0)$ & $1.31(0.05)$ & $6420(220)$ & $34.97(0.07)$ & $34.95(0.07)$ & CSP-I & $\mathrm{PCM} / \mathrm{SCM}$ \\
\hline $2008 \mathrm{gr}^{\mathrm{c}}$ & 0.039 & 0.0229 & $0.0218(0.00015)$ & $54766.5(4.0)$ & $0.91(0.06)$ & $7540(170)$ & $34.42(0.06)$ & $34.73(0.06)$ & CSP-I & $\mathrm{PCM} / \mathrm{SCM}$ \\
\hline $2008 \mathrm{hg}^{\mathrm{c}}$ & 0.050 & 0.0190 & $0.0182(0.00006)$ & $54779.7(5.0)$ & $-1.32(0.56)$ & $4300(180)$ & $34.70(0.20)$ & $34.82(0.08)$ & CSP-I & $\mathrm{PCM} / \mathrm{SCM}$ \\
\hline 2008ho & 0.052 & 0.0103 & $0.0096(0.00050)$ & $54792.7(5.0)$ & $-0.72(0.07)$ & $\ldots$ & $\cdots$ & $\cdots$ & CSP- I & $\mathrm{PCM} / \mathrm{SCM}$ \\
\hline 2008if & 0.090 & 0.0115 & $0.0125(0.00008)$ & $54807.8(5.0)$ & $1.27(0.04)$ & $7100(160)$ & $33.61(0.06)$ & $33.74(0.06)$ & CSP-I & $\mathrm{PCM} / \mathrm{SCM}$ \\
\hline 2008il & 0.045 & 0.0210 & $0.0203(0.00005)$ & $54825.6(3.0)$ & $-0.07(0.07)$ & $\cdots$ & $34.90(0.07)$ & $\cdots$ & CSP-I & $\mathrm{PCM} / \mathrm{SCM}$ \\
\hline 2008in & 0.061 & 0.0050 & $0.0064(0.00008)$ & $54822.8(6.0)$ & $0.03(0.04)$ & $\ldots$ & $\ldots$ & $\ldots$ & CSP-I & $\mathrm{PCM} / \mathrm{SCM}$ \\
\hline $2009 \mathrm{~N}^{\mathrm{c}}$ & 0.057 & 0.0034 & $0.0046(0.00008)$ & $54846.7(5.0)$ & $-0.73(0.03)$ & $\cdots$ & $\cdots$ & $\ldots$ & CSP-I & $\mathrm{PCM} / \mathrm{SCM}$ \\
\hline $2009 \mathrm{ao}^{\mathrm{c}}$ & 0.106 & 0.0111 & $0.0122(0.00007)$ & $54890.6(4.0)$ & $0.93(0.10)$ & $5570(160)$ & $33.61(0.07)$ & $33.42(0.07)$ & CSP-I & $\mathrm{PCM} / \mathrm{SCM}$ \\
\hline $2009 \mathrm{bu}^{\mathrm{c}}$ & 0.070 & 0.0116 & $0.0112(0.00004)$ & $54907.9(6.0)$ & $-0.31(0.03)$ & $5670(160)$ & $33.40(0.07)$ & $33.61(0.07)$ & CSP-I & $\mathrm{PCM} / \mathrm{SCM}$ \\
\hline $2009 \mathrm{bz}^{\mathrm{c}}$ & 0.110 & 0.0108 & $0.0113(0.00004)$ & $54915.8(4.0)$ & $0.07(0.04)$ & $6030(160)$ & $33.67(0.08)$ & $33.92(0.07)$ & CSP-I & $\mathrm{PCM} / \mathrm{SCM}$ \\
\hline 18321 & 0.080 & 0.1041 & $0.1065(0.00050)$ & $54353.6(5.0)$ & $1.34(0.20)$ & $6690(180)$ & $38.09(0.28)$ & $38.93(0.23)$ & SDSS-II & $\mathrm{PCM} / \mathrm{SCM}$ \\
\hline $2006 \mathrm{gq}$ & 0.096 & 0.0697 & $0.0687(0.00050)$ & $53992.4(3.0)$ & $0.28(0.07)$ & $4890(230)$ & $37.34(0.10)$ & $37.56(0.12)$ & SDSS-II & $\mathrm{PCM} / \mathrm{SCM}$ \\
\hline 2006iw & 0.137 & 0.0308 & $0.0295(0.00050)$ & $54010.7(1.0)$ & $0.14(0.05)$ & $6900(510)$ & $35.44(0.08)$ & $35.70(0.12)$ & SDSS-II & $\mathrm{PCM} / \mathrm{SCM}$ \\
\hline 2006j1 & 0.504 & 0.0555 & $0.0554(0.00050)$ & $54006.8(15.0)$ & $0.52(0.07)$ & $8640(700)$ & $\cdots$ & $36.81(0.15)$ & SDSS-II & $\mathrm{PCM} / \mathrm{SCM}$ \\
\hline $2006 \mathrm{kn}$ & 0.194 & 0.1199 & $0.1190(0.00050)$ & $54007.0(1.5)$ & $1.55(0.36)$ & $6330(200)$ & $38.43(0.29)$ & $38.48(0.25)$ & SDSS-II & $\mathrm{PCM} / \mathrm{SCM}$ \\
\hline $2006 \mathrm{kv}$ & 0.080 & 0.0619 & $0.0620(0.00050)$ & $54016.5(4.0)$ & $1.60(0.23)$ & $5590(190)$ & $37.26(0.14)$ & $37.08(0.14)$ & SDSS-II & $\mathrm{PCM} / \mathrm{SCM}$ \\
\hline $2007 \mathrm{kw}$ & 0.074 & 0.0681 & $0.0672(0.00050)$ & $54361.6(2.5)$ & $1.00(0.08)$ & $6610(250)$ & 37.02(0.09) & $37.10(0.09)$ & SDSS-II & $\mathrm{PCM} / \mathrm{SCM}$ \\
\hline 2007ky & 0.105 & 0.0737 & $0.0727(0.00050)$ & $54363.5(3.0)$ & $0.38(0.37)$ & $5170(170)$ & $37.62(0.16)$ & $37.17(0.14)$ & SDSS-II & $\mathrm{PCM} / \mathrm{SCM}$ \\
\hline $2007 \mathrm{kz}$ & 0.320 & 0.1275 & $0.1270(0.00050)$ & $54362.6(3.5)$ & $1.44(0.33)$ & $6060(200)$ & $38.71(0.25)$ & $39.02(0.33)$ & SDSS-II & $\mathrm{PCM} / \mathrm{SCM}$ \\
\hline 2007lb & 0.496 & 0.0379 & $0.0375(0.00050)$ & $54368.8(7.0)$ & $0.22(0.07)$ & $7350(370)$ & $35.31(0.07)$ & $35.77(0.10)$ & SDSS-II & $\mathrm{PCM} / \mathrm{SCM}$ \\
\hline 2007ld & 0.255 & 0.0270 & $0.0267(0.00500)$ & $54369.6(5.5)$ & $0.53(0.05)$ & $6620(420)$ & $35.07(0.07)$ & $35.24(0.42)$ & SDSS-II & $\mathrm{PCM} / \mathrm{SCM}$ \\
\hline $20071 \mathrm{j}$ & 0.118 & 0.0500 & $0.0489(0.00500)$ & $54370.2(3.5)$ & $0.84(0.06)$ & $5610(300)$ & $36.72(0.07)$ & $36.43(0.25)$ & SDSS-II & $\mathrm{PCM} / \mathrm{SCM}$ \\
\hline 2007 lx & 0.120 & 0.0571 & $0.0559(0.00050)$ & $54374.5(8.0)$ & $0.47(0.11)$ & $5520(200)$ & $37.07(0.22)$ & $37.10(0.11)$ & SDSS-II & $\mathrm{PCM} / \mathrm{SCM}$ \\
\hline $2007 \mathrm{nr}$ & 0.079 & 0.1400 & $0.1389(0.00050)$ & $54353.5(5.0)$ & $1.25(0.46)$ & $5230(190)$ & $39.53(0.40)$ & $39.27(0.30)$ & SDSS-II & $\mathrm{PCM} / \mathrm{SCM}$ \\
\hline 2007nw & 0.204 & 0.0571 & $0.0555(0.00050)$ & $54372.2(7.0)$ & $-0.06(0.17)$ & $5810(200)$ & $37.17(0.12)$ & $36.96(0.12)$ & SDSS-II & $\mathrm{PCM} / \mathrm{SCM}$ \\
\hline 2007ny & 0.080 & 0.1429 & $0.1419(0.00050)$ & $54367.7(7.0)$ & $1.39(1.44)$ & $6860(190)$ & $39.43(0.64)$ & $39.14(0.45)$ & SDSS-II & $\mathrm{PCM} / \mathrm{SCM}$ \\
\hline 03D1bo & 0.066 & 0.3279 & $0.3271(0.00100)$ & $52888.0(4.0)$ & $\ldots$ & $\ldots$ & $\ldots$ & $\ldots$ & SNLS & $\mathrm{PCM} / \mathrm{SCM}$ \\
\hline 03D1bz & 0.066 & 0.2939 & $0.2931(0.00100)$ & $52914.0(4.0)$ & $0.16(0.23)$ & $\cdots$ & $\cdots$ & $\cdots$ & SNLS & $\mathrm{PCM} / \mathrm{SCM}$ \\
\hline 03D3ce & 0.026 & 0.2880 & $0.2884(0.00100)$ & $52780.0(10.0)$ & $\cdots$ & $\cdots$ & $\cdots$ & $\cdots$ & SNLS & $\mathrm{PCM} / \mathrm{SCM}$ \\
\hline 03D4az & 0.076 & 0.4079 & $0.4069(0.00100)$ & $52808.0(4.0)$ & $-0.47(1.49)$ & $\ldots$ & $\ldots$ & $\ldots$ & SNLS & PCM \\
\hline 03D4bl & 0.072 & 0.3179 & $0.3169(0.00100)$ & $52822.0(3.0)$ & $1.32(1.46)$ & $\ldots$ & $41.43(0.57)$ & $\ldots$ & SNLS & PCM \\
\hline 03D4da & 0.078 & 0.3279 & $0.3269(0.00100)$ & $52874.0(7.0)$ & $\ldots$ & $\ldots$ & $\ldots$ & $\ldots$ & SNLS & $\mathrm{PCM} / \mathrm{SCM}$ \\
\hline 04D1ha & 0.073 & 0.4839 & $0.4831(0.00100)$ & $53233.0(3.0)$ & $0.11(0.42)$ & $\ldots$ & $42.13(0.44)$ & $\ldots$ & SNLS & PCM \\
\hline 04D1ln & 0.071 & 0.2069 & $0.2062(0.00100)$ & $53274.0(5.0)$ & $0.32(0.12)$ & $\cdots$ & $40.28(0.11)$ & $\cdots$ & SNLS & $\mathrm{PCM} / \mathrm{SCM}$ \\
\hline 04D1nz & 0.072 & 0.2629 & $0.2621(0.00100)$ & $53264.0(4.0)$ & $0.13(0.37)$ & $\cdots$ & 40.74(0.30) & $\cdots$ & SNLS & PCM \\
\hline 04D1pj & 0.076 & 0.1559 & $0.1552(0.00100)$ & $53304.0(8.0)$ & $0.02(0.14)$ & $5975(230)$ & $39.13(0.09)$ & $39.37(0.10)$ & SNLS & $\mathrm{PCM} / \mathrm{SCM}$ \\
\hline 04D1qa & 0.072 & 0.1719 & $0.1711(0.00100)$ & $53300.0(3.0)$ & $-0.10(0.40)$ & $\ldots$ & $39.65(0.19)$ & $\ldots$ & SNLS & PCM \\
\hline 04D2dc & 0.053 & 0.1849 & $0.1861(0.00100)$ & $53040.0(25.0)$ & $0.14(0.19)$ & $\cdots$ & $\ldots$ & $\ldots$ & SNLS & $\mathrm{PCM} / \mathrm{SCM}$ \\
\hline 04D4fu & 0.072 & 0.1329 & $0.1319(0.00100)$ & $53213.0(6.0)$ & $0.23(0.60)$ & $4785(200)$ & $39.21(0.23)$ & $39.02(0.10)$ & SNLS & $\mathrm{PCM} / \mathrm{SCM}$ \\
\hline
\end{tabular}




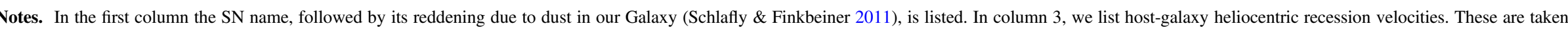

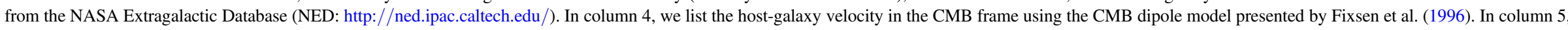

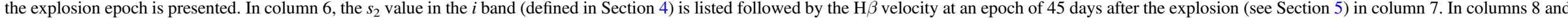

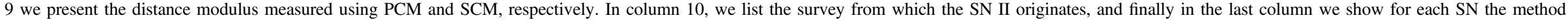
available, i.e., whether a spectrum is available or not.

a 45 days post-explosion.

${ }^{\mathrm{b}}$ CSP-I = Carnegie Supernova Project-I, SDSS-II = Sloan Digital Sky Survey II SN, SNLS = Supernova Legacy Survey.

${ }^{c}$ SNe II used in de Jaeger et al. (2015). 
The images were obtained using the wide-field SDSS-II CCD camera (Gunn et al. 1998), and the photometry was computed using the five ugriz filters defined in Fukugita et al. (1996). More information about the data reduction can be found in York et al. (2000), Ivezić et al. (2004), and Holtzman et al. (2008).

A spectroscopic follow-up program was performed and uncertainties derived on the redshift measurement are about 0.0005 when the redshift is measured using the host-galaxy spectra, and about 0.005 when the $\mathrm{SN}$ spectral features are used.

The total SDSS-II SN sample is composed of 16 spectroscopically confirmed $\mathrm{SNe}$ II of which $15 \mathrm{SNe}$ II are from D'Andrea et al. (2010), and we add one SN II (SN 2007ny) removed by D'Andrea et al. (2010) from his SCM sample due to the absence of explosion date estimation. We derive an explosion date using the González-Gaitán et al. (2015) rise model. From the SDSS-II sample, we exclude SN 2007nv due to its large $i$-band uncertainties (D'Andrea et al. 2010). Note that the majority of spectra were obtained soon after explosion, and therefore they only clearly exhibit $\mathrm{H}_{\alpha} \lambda 6563$ and $\mathrm{H}_{\beta} \lambda 4861$ lines but very weak Fe II $\lambda 5018$ or Fe II $\lambda 5169$ lines, which are often used for the SCM.

\subsection{Supernova Legacy Survey}

In order to obtain a more complete Hubble diagram, we also use higher redshift SNe II from the SNLS. The SNLS was designed to discover $\mathrm{SNe}$ and to obtain photometric follow-up using the MegaCam imager (Boulade et al. 2003) on the $3.6 \mathrm{~m}$ Canada-France-Hawaii Telescope. The observation strategy consisted of obtaining images of the same field every four nights over five years (between 2003 and 2008); thus, in total more than 470 nights were allocated to this project. Even though the sample was designed for SNe Ia cosmology and was very successful (Guy et al. 2010; Conley et al. 2011; Sullivan et al. 2011; Betoule et al. 2014), the observation of many SNe II with $0.1 \leqslant z \leqslant 0.5$, with good explosion date constraints and good photometric coverage allowed the use of this sample to construct an SN II Hubble diagram (Nugent et al. 2006), to constrain SN II rise times (González-Gaitán et al. 2015), and to derive a precise measurement of the corecollapse SN rate (Bazin et al. 2009).

Photometry was obtained in four pass bands $(g, r, i, z)$ similar to those used by SDSS-II and CSP-I (Regnault et al. 2009). After each run, the images were pre-processed using the Elixir pipeline (Magnier \& Cuillandre 2004) and then, sky background subtraction, astrometry, and photometric correction were performed using two different and independent pipelines. The description of all the data reduction steps can be found in Astier et al. (2006), Baumont et al. (2008), Regnault et al. (2009), Guy et al. (2010), Perrett et al. (2010), and Conley et al. (2011).

Due to the redshift of the $\mathrm{SNe}$ and their faintness, spectroscopy was obtained using different large telescopes. All spectra were reduced in a standard way as described in Howell et al. (2005), Bronder et al. (2008), Ellis et al. (2008), Balland et al. (2009), and Walker et al. (2011).

We select only SNe II from the full photometric sample (more than 6000 objects), in particular SNe II with spectroscopic redshift from the $\mathrm{SN}$ or the host, a spectroscopic classification, or a good photometric classification (based on the Gonzalez method described in Kessler et al. 2010), and a well-defined explosion date, as done by González-Gaitán et al. (2015). The total SNLS sample is composed of 28 SNe II, 4 of them were used in Nugent et al. (2006) to derive the first SNe II high redshift Hubble diagram. For this sample, $16 \mathrm{SNe}$ II have a spectrum and could potentially be used for the SCM. SN 07D2an was identified as an SN 1987A-like event by González-Gaitán et al. (2015) and is removed from the SNLS sample. As for the SDSS-II sample, the majority of the spectra do not show clear Fe II $\lambda 5018$ or Fe II $\lambda 5169$ absorption lines, which prevented us from measuring the photospheric expansion velocities using these lines. Fortunately, many SNe II also exhibit a strong $\mathrm{H}_{\beta}$ absorption line $(\lambda 4861)$, which is useful for the SCM.

\section{Methodology}

\subsection{Background}

The photon flux observed in one photometric system is affected by four different sources: the dust in our Milky Way (AvG), the expansion of the universe (K-correction; Oke \& Sandage 1968; Hamuy et al. 1993; Kim et al. 1996; Nugent et al. 2002), the host-galaxy extinction (Avh), and by the difference between the natural photometric system used to obtain observations and the standard photometric system (Scorrection; Stritzinger et al. 2002). In the following we describe how we account for these factors and place all of the photometry on a common photometric system.

Correcting for $\mathrm{AvG}$ is straightforward using the value derived by Schlafly \& Finkbeiner (2011) and gathered in the NASA/IPAC Extragalactic Database $\left(\mathrm{NED}^{24}\right)$. Correcting for Avh is more complicated. To date, no accurate methods exist. For example, the equivalent width of the $\mathrm{Na}$ I doublet lines (Turatto et al. 2003) is a bad proxy for extinction (Poznanski et al. 2011). For the color-color diagram and multiband fit methods (Folatelli et al. 2013; Rodríguez et al. 2014), better understanding and estimation of the SNe II intrinsic color are necessary to derive a good approximation of Avh. To take into account the host-galaxy extinction, similarly to what has been done in $\mathrm{SNe}$ Ia cosmology, we add a color term correction in the standardization method, which takes care of the magnitudecolor variations independently of their origin. The AvG, K-, and S-corrections (AKS) are finally simultaneously computed using the cross-filter K-corrections defined by Kim et al. (1996) from an observed filter (CSP-I, SDSS-II, or SNLS) and the standard system. The CSP-I natural system was chosen as the common photometric system, and thus we transform all the photometry to the CSP-I system. The AKS correction depends on anything that could affect the SED (spectral energy distribution) continuum; it is thus very important to adjust the continuum to have the same color as the SN II through a color-matching function (Nugent et al. 2002; Hsiao et al. 2007). In Section 3.2 we describe the procedure for applying the AKS correction.

\subsection{Procedure}

In practice, to apply the AKS correction, a SED template series is needed. We adopt a sequence of theoretical spectral models from Dessart et al. (2013), consisting of an SN

\footnotetext{
${ }^{4}$ NASA/IPAC Extragalactic Database (NED) is operated by the Jet Propulsion Laboratory, California Institute of Technology, under contract with the National Aeronautics and Space Administration.
} 
progenitor with a main-sequence mass of $15 M_{\odot}$, solar metallicity $Z=0.02$, zero rotation, and a mixing-length parameter of 3 . The choice of model was motivated by the very good match of the theoretical model to the data of the prototypical SNe II (SN 1999em). We describe step by step the method to transform an observed magnitude to the CSP-I photometric system. The first two steps can be found in de Jaeger et al. (2015) and consist of selecting the theoretical spectrum that has an epoch closest in time to the respective epoch of the observed light-curve point of the $\mathrm{SN}$ in consideration and bringing it to the observed frame using the heliocentric redshift.

1. We adjust the SED continuum to match the observed color by comparing synthetic magnitudes with observed magnitudes using the zero points defined in Fukugita et al. (1996), Stritzinger et al. (2002), and Regnault et al. (2009). A color-matching (CM) function $\mathrm{CM}(\lambda)$ is obtained and used to correct our model spectrum. Finally, we obtain $f_{\mathrm{CM}}^{\text {obs }}(\lambda)=\mathrm{CM}(\lambda) \times f^{\text {obs }}(\lambda)$ and calculate the magnitude in the observer frame:

$$
m_{Y}=-2.5 \log _{10}\left[\int f_{\mathrm{CM}}^{\mathrm{obs}}(\lambda) S_{Y} \lambda d \lambda\right]+Z P_{Y},
$$

with $\lambda$ the wavelength, $S_{Y}$ the transmission function of filter $Y$ (CSP-I, SDSS-II, or SNLS), and $Z P_{Y}$ the zero point of filter $Y$.

2. Using a Cardelli et al. (1989) law, we correct for AvG and bring the unredenned spectrum to the rest-frame $f_{\mathrm{CM}}^{\text {rest, } \mathrm{AvG}}\left(\lambda^{\prime}\right)$. The magnitude $X$ in the rest-frame is computed using

$$
\begin{aligned}
m_{X}^{\mathrm{CSP}-I}= & -2.5 \log _{10} \int f_{\mathrm{CM}}^{\mathrm{rest}, \mathrm{AvG}}(\lambda) S_{X}^{\mathrm{CSP}-I} \lambda d \lambda \\
& +Z P_{X}^{\mathrm{CSP}-I},
\end{aligned}
$$

where $S_{X}^{\mathrm{CSP}-I}$ is the transmission function of filter $X$ and $Z P_{X}^{\mathrm{CSP}-I}$ is the zero point of filter $X$ of the CSP-I system (see Contreras et al. 2010; Stritzinger et al. 2011). The choice of filter $X$ depends on the redshift. At low redshift, the $\mathrm{K}$-correction is not that important, so $X$ and $Y$ are the same bands, but at higher redshifts this is not the case. We need to know in which band a photon received in the $Y$ band has been emitted. For this, we calculate the effective wavelength of the filter $Y$ corrected by the $\left(1+z_{\text {hel }}\right)$ factor and select the closest effective wavelength among the CSP-I filters $(u, g, r, i)$. Then the AKS is obtained from

$$
\mathrm{AKS}_{X Y}=m_{Y}-m_{X}^{\mathrm{CSP}-I} .
$$

The AKS corrections are sensitive to the choice of spectral template; thus, in order to estimate the systematic errors, we try another model: Nugent's templates. ${ }^{25}$ These templates are based on the models from Baron et al. (2004). The comparison between Nugent's templates and Dessart's model (Dessart et al. 2013) leads to a mean difference of 0.004 mag in the $r$ band (with a standard deviation of $0.02 \mathrm{mag}$ ) and $0.03 \mathrm{mag}$ (with a standard deviation of $\pm 0.06 \mathrm{mag}$ ) in the $i$ band. It is important to note that both models were created to fit the same observed data (SN 1999em spectra) and a comparison between

\footnotetext{
25 https://c3.lbl.gov/nugent/index.html
}

Dessart's model and the observed spectra using the CSP-I sample was achieved in de Jaeger et al. (2015), who derived a good agreement.

\section{The Photometric Color Method (PCM)}

\subsection{Methodology}

The basic idea of this method is to correct and standardize the apparent magnitude using two photometric parameters: $s_{2}$, which is the slope of the plateau, and a color term at a specific epoch (de Jaeger et al. 2015). To measure $s_{2}$ in all the bands, we use a Python program, which consists of performing a leastsquares fitting of the AKS-corrected light curves, with one and two slopes (sometimes the first decline after the maximum is not visible). To choose between one or two slopes, the statistical method F-test is performed. The slope is measured in each band $(g, r$, and $i)$ but only the values used in this work $(i$ band) are listed in Table 1. The minimization of intrinsic dispersion in the Hubble diagram is our figure of merit and allows us to find the best combination possible of filter, color, and epoch as done by de Jaeger et al. (2015). Note that using only the CSP-I sample, we derive the $V$-band light curve slopes and perform a sanity check by comparing these values with those found by Anderson et al. (2014). We obtain a very good agreement. Also, using 51 SNe II, Galbany et al. (2016) confirmed the relation found by Anderson et al. (2014) between $s_{2}$ and the absolute magnitude in different bands. The observed magnitudes can be modeled as

$$
\begin{aligned}
m_{\lambda 1}^{\text {model }}= & M_{\lambda 1}-\alpha s_{2}+\beta_{\lambda 1}\left(m_{\lambda 2}-m_{\lambda 3}\right) \\
& +5 \log _{10}\left(d_{L}\left(z_{\mathrm{CMB}} \mid \Omega_{m}, \Omega_{\Lambda}\right)\right)+25,
\end{aligned}
$$

where $\left(m_{\lambda 2}-m_{\lambda 3}\right)$ is the color, $d_{L}\left(z_{\mathrm{CMB}} \mid \Omega_{m}, \Omega_{\Lambda}\right)$ is the luminosity distance for a cosmological model depending on the cosmological parameters $\Omega_{m}, \Omega_{\Lambda}$; the CMB redshift $z_{\mathrm{CMB}}$; and the Hubble constant. Finally, $\alpha, \beta_{\lambda_{1}}$, and $M_{\lambda_{1}}$ are also free parameters, with $M_{\lambda_{1}}$ corresponding to the absolute magnitude in the filter $\lambda_{1}$. Note that $s_{2}$ and the color distributions are centered, i.e., we use $\left(s_{2}-\left\langle s_{2}\right\rangle\right)$ and $\left(m_{\lambda 2}-m_{\lambda 3}\right)-\left\langle m_{\lambda 2}-m_{\lambda 3}\right\rangle$, where $\left\langle s_{2}\right\rangle$ and $\left\langle m_{\lambda 2}-m_{\lambda 3}\right\rangle$ are the mean values of the slope and the color respectively for the entire sample (CSP-I+SDSSII + SNLS).

Since we do not have in our sample any SN II with an accurate distance estimation (e.g., from Cepheid measurements), we only measure relative distances and define the "Hubble Constant free" absolute magnitude as $\mathcal{M}_{\lambda 1}=\mathrm{M}_{\lambda 1}-5$ $\log _{10}\left(\mathrm{H}_{0}\right)+25$ and $\mathcal{D}_{L}=\mathrm{H}_{0} d_{L}$ as done in many previous works (Perlmutter et al. 1999; Nugent et al. 2006; Poznanski et al. 2009; D'Andrea et al. 2010). The apparent magnitude is finally written as

$$
\begin{aligned}
m_{\lambda 1}^{\text {model }}= & \mathcal{M}_{\lambda 1}-\alpha s_{2}+\beta_{\lambda 1}\left(m_{\lambda 2}-m_{\lambda 3}\right) \\
& +5 \log _{10}\left(\mathcal{D}_{L}\left(z_{\mathrm{CMB}} \mid \Omega_{m}, \Omega_{\Lambda}\right)\right) .
\end{aligned}
$$

From this equation, one can derive $\alpha, \beta_{\lambda_{1}}, \mathcal{M}_{\lambda 1}$, and the cosmological parameters $\Omega_{m}, \Omega_{\Lambda}$. Due to our imperfect knowledge of SN II physics, another free parameter named $\sigma_{\text {int }}$ needs to be added in order to include the intrinsic scatter not accounted for in the measurement errors. This dispersion is the minimum statistical uncertainty in any distance determination using PCM.

In cosmology, when we compare observations to predictions of a parameter-dependent model, Bayesian inference is the 
standard procedure. This approach tells us how to update our knowledge from a "prior" distribution to a new probability density named "posterior." Thus, to define the posterior probability density one needs to define a likelihood function and a prior function. As the likelihood function, we choose that defined by D'Andrea et al. (2010):

$$
-2 \ln (\mathcal{L})=\sum_{\mathrm{SN}}\left\{\frac{\left[m_{\lambda 1}^{\mathrm{obs}}-m_{\lambda 1}^{\text {model }}\right]^{2}}{\sigma_{\text {tot }}^{2}}+\ln \left(\sigma_{\text {tot }}^{2}\right)\right\},
$$

where we sum over all SNe II available for one specific epoch, $\mathrm{m}_{\lambda 1}^{\mathrm{obs}}$ is the observed magnitude corrected for AKS, $\mathrm{m}_{\lambda 1}^{\text {model }}$ is the model defined in Equation (5), and the total uncertainty $\sigma_{\text {tot }}$, corresponding to the error propagation of the model, is defined as

$$
\begin{aligned}
\sigma_{\text {tot }}^{2}= & \sigma_{m_{\lambda 1}}^{2}+\left(\alpha \sigma_{s 2}\right)^{2}+\left(\beta \sigma_{\left(m_{\lambda 2}-m_{\lambda 3}\right)}\right)^{2} \\
& +\left(\sigma_{z} \frac{5(1+z)}{z(1+z / 2) \ln (10)}\right)^{2}+\sigma_{\text {int }}^{2} .
\end{aligned}
$$

For the relation between the redshift uncertainty and the associated magnitude uncertainties, we use the empty universe approximation (Conley et al. 2011). The second logarithmic term comes from the normalization of the likelihood function and is useful in order to not obtain large values of $\alpha, \beta$, and $\mathcal{M}_{\lambda 1}$, which could be favored by the first part of the loglikelihood.

Our prior probability distribution is defined to have uniform probability for $0 \leqslant \Omega_{m} \leqslant 1$ or $\alpha, \beta, \mathcal{M}_{\lambda 1} \neq 0$, but otherwise has zero probability. We also attempted to use a Gaussian prior, however no differences were found in the fit parameters. To explore the posterior probability density, a Monte Carlo Markov Chain (MCMC) simulation is performed. The MCMC calculation is run using a Python package called EMCEE developed by Foreman-Mackey et al. (2013) and using 500 walkers and 1000 steps (for the convergence, as suggested by the authors of the EMCEE package, we checked the fraction acceptance, which should be between 0.2 and 0.5 ). The EMCEE package uses an ensemble of walkers that can be moved in parallel and not a single iterative random walker (the Goodman-Weare algorithm versus the Metropolis-Hastings algorithm).

The entire sample available for this method contains 105 SNe II. From this sample, in order to avoid peculiar galaxy motions, we select only the SNe II with $c z_{\mathrm{CMB}} \geqslant 3000 \mathrm{~km} \mathrm{~s}^{-1}$. After this first cut, the sample size drops to 89 SNe II including $45 \mathrm{SNe}$ II from CSP-I, $16 \mathrm{SNe}$ II from SDSS-II, and $28 \mathrm{SNe}$ II from SNLS.

\subsection{Results}

In this results section, we will first attempt to extend the lowredshift PCM Hubble diagram (de Jaeger et al. 2015) to higher redshifts, and then investigate whether such efforts can constrain cosmological parameters (Section 4.2.2).

\subsubsection{Fixed Cosmology}

In order to test the method at higher redshifts, we first assume a fiducial $\Lambda \mathrm{CDM}$ model, i.e., a flat universe $\left(\Omega_{m}+\Omega_{\Lambda}=1\right)$ with $\Omega_{m}=0.3$ and $\Omega_{\Lambda}=0.7$. We assume also a Hubble constant of $70 \mathrm{~km} \mathrm{~s}^{-1} \mathrm{Mpc}^{-1}$ because we are not

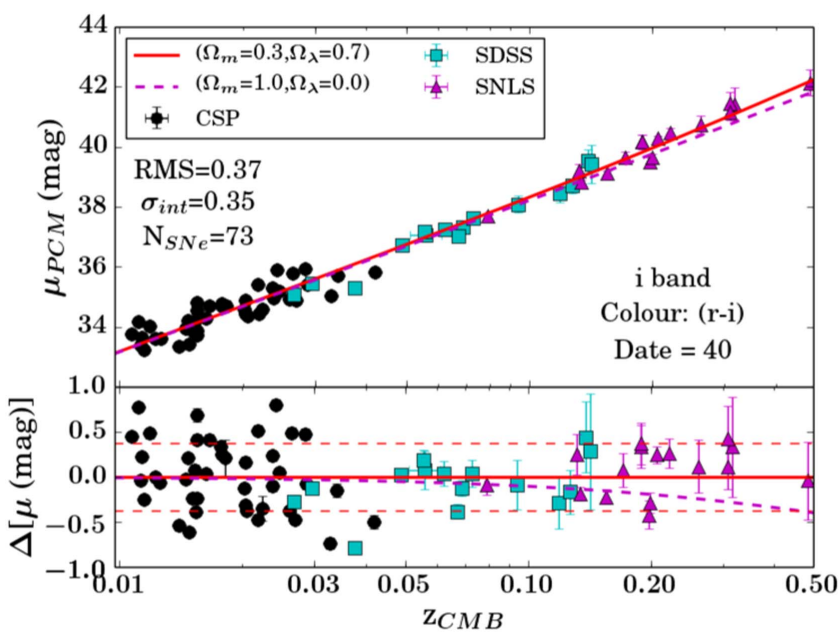

Figure 1. Hubble diagram for SNe II, using the PCM and all of the SNe II available at this epoch from the CSP-I, SDSS-II, and SNLS samples, respectively. Black dots represent the SNe II from CSP-I whereas the cyan squares and magenta triangles are those from the SDSS-II and the SNLS samples, respectively. The red line is the Hubble diagram for the $\Lambda \mathrm{CMB}$ $\left(\Omega_{m}=0.3\right.$ and $\left.\Omega_{\Lambda}=0.7\right)$ and the magenta line is for an Einstein-de Sitter cosmological model $\left(\Omega_{m}=1.0\right.$ and $\left.\Omega_{\Lambda}=0.0\right)$. In both models, we assume a Hubble constant of $70 \mathrm{~km} \mathrm{~s}^{-1} \mathrm{Mpc}^{-1}$. In the bottom panel, the residuals with respect to the $\Lambda \mathrm{CMB}$ are shown. We also present the number of SNe II available at this epoch $\left(N_{\mathrm{SNe}}\right)$, the epoch after the explosion (Date), the root mean square (rms), and the intrinsic dispersion $\left(\sigma_{\text {int }}\right)$.

able to derive a Hubble constant using our low-redshift sample (lack of SNe II with Cepheid measurements).

Using all the filter combinations available for the three surveys $(g, r, i)$, we find a minimum intrinsic dispersion for the $(r-i)$ color associated with the $i$ band for an epoch of rest-frame day 40. At this specific epoch, only 73 SNe II have photometric data. In this work, we interpolate all the magnitudes and colors at this epoch but we do not extrapolate, leaving us with only 73 $\mathrm{SNe}$ II from the entire sample (89 SNe II). In Figure 1, we present the final Hubble diagram using the PCM. For the 73 $\mathrm{SNe}$ II in the Hubble flow available at this epoch and at this specific color, we obtain an intrinsic scatter of 0.35 mag, i.e., $16 \%$ in distance errors. The use of the PCM allows us to reduce the intrinsic scatter from $0.57 \mathrm{mag}$ (raw magnitudes) to 0.35 mag, i.e., an improvement of $10 \%$ in distance errors. This scatter is somewhat lower than that found by de Jaeger et al. (2015), due to the higher redshift SNe II (0.4-0.44 mag), which, as will be shown in Section 6.1, exhibit a smaller range in absolute magnitude. The Bayesian inference procedure using the likelihood defined in Equation (6) gives $\alpha=0.36_{-0.06}^{+0.06}$, $\beta=0.70_{-0.29}^{+0.29}$, and $\mathcal{M}_{\lambda 1}=-1.09_{-0.05}^{+0.05}$. Using only the CSP-I sample as done in de Jaeger et al. (2015), we find $\alpha=0.39_{-0.08}^{+0.08}, \beta=0.80_{-0.48}^{+0.47}$, and $\mathcal{M}_{\lambda 1}=-1.06_{-0.07}^{+0.06}$. These values are consistent with those derived by de Jaeger et al. (2015). From $\mathcal{M}_{\lambda 1}$ and with $H_{0}=70 \mathrm{~km} \mathrm{~s}^{-1} \mathrm{Mpc}^{-1}$, an absolute magnitude $M_{i}$ of $-16.84_{-0.06}^{+0.06} \mathrm{mag}$ is obtained. This value is relatively low compared to the value reported by Richardson et al. (2014); we do not account for the intrinsic color $\left(m_{\lambda 2}-m_{\lambda 3}\right)_{\text {int }}$, where "int" is for intrinsic. Indeed, in our model (Equation (5)), the host-galaxy extinction is taken into account using the observed color; however, only the excess color $\left(E\left(m_{\lambda 2}-m_{\lambda 3}\right)\right)$ is directly related to Avh and should be used. In the following equations, we show the relation between 
Table 2

PCM-fit Parameters

\begin{tabular}{lccccc}
\hline \hline Data Set & $\alpha$ & $\beta$ & $M_{i}$ & $\sigma_{\text {int }}$ & SNe \\
\hline CSP-I & $0.39_{-0.08}^{+0.08}$ & $0.80_{-0.48}^{+0.47}$ & $-16.84_{-0.07}^{+0.06}$ & $0.41_{-0.04}^{+0.05}$ & 42 \\
CSP-I+SDSS-II & $0.38_{-0.07}^{+0.07}$ & $0.75_{-0.36}^{+0.36}$ & $-16.87_{-0.05}^{+0.05}$ & $0.38_{-0.04}^{+0.04}$ & 57 \\
CSP-I+SNLS & $0.36_{-0.07}^{+0.07}$ & $0.87_{-0.35}^{+0.34}$ & $-16.83_{-0.05}^{+0.05}$ & $0.37_{-0.03}^{+0.04}$ & 58 \\
SDSS-II+SNLS & $0.28_{-0.10}^{+0.10}$ & $0.69_{-0.36}^{+0.36}$ & $-16.90_{-0.06}^{+0.06}$ & $0.27_{-0.04}^{+0.05}$ & 31 \\
CSP-I+SDSS- & $0.36_{-0.06}^{+0.06}$ & $0.71_{-0.28}^{+0.29}$ & $-16.85_{-0.05}^{+0.05}$ & $0.36_{-0.03}^{+0.03}$ & 73 \\
$\quad$ II+SNLS & & & & & \\
\hline
\end{tabular}

Note. Best-fit values and the associated errors for each parameter for different samples using the PCM.

the Avh, the excess color, and the intrinsic color:

$$
\begin{aligned}
A_{\lambda 1} & =\beta_{\lambda 1} \times E\left(m_{\lambda 2}-m_{\lambda 3}\right) \\
& =\beta_{\lambda 1} \times\left(m_{\lambda 2}-m_{\lambda 3}\right)-\beta_{\lambda 1}\left(m_{\lambda 2}-m_{\lambda 3}\right)_{\mathrm{int}} \\
& =\beta_{\lambda 1} \times\left(m_{\lambda 2}-m_{\lambda 3}\right)+\text { constant. }
\end{aligned}
$$

Thus, the intrinsic color is degenerate with $\mathcal{M}_{\lambda 1}$, so any approximation (we assume that the intrinsic color is zero) on this value has consequences for the absolute magnitude determination.

Systematic errors coming from the SN II sample at different redshifts are investigated by looking at the evolution of the fitting parameters using different samples, i.e., CSP-I, CSP-I +SDSS-II, CSP-I+SNLS, SDSS-II+SNLS, and CSP-I + SDSS-II+SNLS. In Table 2 a summary of these values is shown. As seen from this table, the fitting parameters remain similar within the uncertainties for the different samples, which means that there does not seem to be a systematic redshift or SNe II sample evolution. We can also study how the parameters are affected by photometric errors. If we arbitrarily increase the centered color distribution by an offset $(0.01 \mathrm{mag}$ and $0.5 \mathrm{mag}$ ), almost all of the fitting parameters remain similar. Only the Hubble constant free absolute magnitude $\mathcal{M}_{\lambda 1}$ changes from to -1.09 to -1.49 , but this is explained by the fact that $\mathcal{M}_{\lambda 1}$ and the intrinsic color are degenerate.

A residual analysis between the data and the $\Lambda \mathrm{CDM}$ cosmological model is also performed by testing for normality (cf. Anderson-Darling test, Stephen 1974), autocorrelation (Durbin-Watson test, Durbin \& Watson 1950), stationarity (Kwiatkowski-Phillips-Schmidt-Shin test; Kwiatkowski et al. 1992), and outliers (Chauvenet's criterion; Chauvenet 1863 , p. 474). For the normality test, at significance levels from $1 \%$ to $15 \%$, we cannot reject the null hypothesis that the residuals come from a Gaussian distribution. In the same way, we cannot reject the null hypothesis of stationarity. Additionally, we do not find existence of autocorrelation and no value should be eliminated according to Chauvenet's criterion.

\subsection{2. $\Omega_{m}$ Derivation}

In this section, we try to put some constraints on the cosmological parameters $\left(\Omega_{m}\right.$ and $\left.\Omega_{\Lambda}\right)$ assuming a Hubble constant $H_{0}=70 \mathrm{~km} \mathrm{~s}^{-1} \mathrm{Mpc}^{-1}$. Due to the lack of higher redshift SNe II (only one with $\mathrm{z} \geqslant 0.4$ ), it is difficult to differentiate between cosmological models, and thus to derive a meaningful constraint on cosmology. Indeed, keeping $\Omega_{m}, \Omega_{\Lambda}$ as free parameters in Equation (5), we are not able to obtain constraints with reasonable error bars. So, we assume a flat universe, i.e., $\Omega_{m}+\Omega_{\Lambda}=1$ and leave only $\Omega_{m}$ as a free parameter. Figure 2 shows a corner plot with all of the one- and

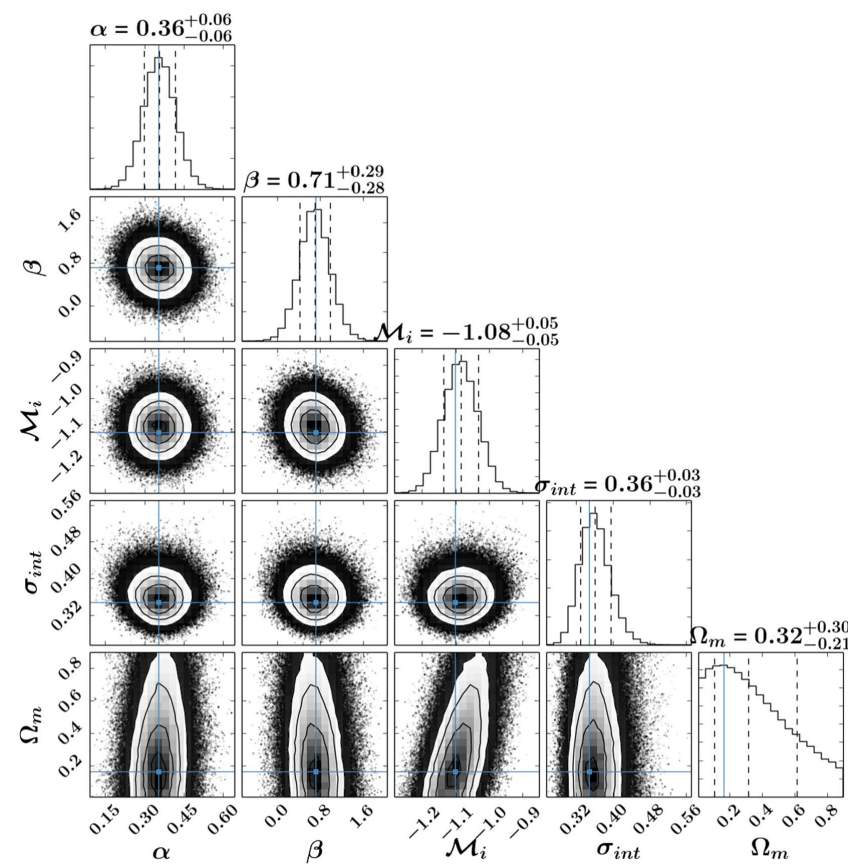

Figure 2. PCM: corner plot showing all of the one- and two-dimensional projections. The blue lines are the values obtained using only one likelihood minimization. Contours are shown at $1 \sigma, 2 \sigma$, and $3 \sigma$. The five free parameters are plotted: $\alpha, \beta, \mathcal{M}_{\lambda 1}, \sigma_{\text {int }}$, and $\Omega_{m}$. To make this figure we use the corner plot package (triangle.py v0.1.1. Zenodo. 10.5281/zenodo.11020).

two-dimensional projections for the five free parameters: $\alpha, \beta$, $\mathcal{M}_{\lambda 1}, \sigma_{\text {int }}$, and $\Omega_{m}$. The first four parameters are defined by a Gaussian distribution (top figure of each column) with small error bars. The values derived are consistent with that found with a fixed cosmology: $\alpha=0.36_{-0.06}^{+0.06}, \quad \beta=0.71_{-0.28}^{+0.29}$, $\mathcal{M}_{\lambda 1}=-1.08_{-0.05}^{+0.05}$, and $\sigma_{\text {int }}=0.36_{-0.03}^{+0.03}$.

For the matter density, the distribution does not look like a Gaussian distribution but the distribution width decreases as the matter density value increases. A value for the matter density of $\Omega_{m}=0.32_{-0.21}^{+0.30}$ is derived, which gives a dark energy density of $\Omega_{\lambda}=0.68_{-0.30}^{+0.21}$. These values are consistent with the $\Lambda \mathrm{CDM}$ cosmological model with uncertainties far from the precision achieved recently using $\mathrm{SNe} \mathrm{Ia}, \Omega_{m}=0.295 \pm 0.034$ (from Betoule et al. 2014 with $\sim 740$ SNe Ia up to a redshift of 1.2; see also Rest et al. 2014 and Scolnic et al. 2014 for other results). However, these errors are comparable to those found by Perlmutter et al. (1997) for which the authors, using $\sim 20$ SNe Ia (7 SNe Ia with $z$ between 0.3 and 0.5 ), derived an uncertainty in the matter density $\Delta \Omega_{m} \sim 0.30$. Note that the minimization of the negative likelihood defined in Equation (6) is found for a value $\Omega_{m}=0.17 \pm 0.30$ (blue line in Figure 2). To test the sensitivity of $\Omega_{m}$ and its uncertainty to the systematic errors, we double the errors on $s_{2}$. The minimum of the negative $\log$ of the likelihood function is obtained for the universe's matter density, 0.22 instead of 0.17 . Using the MCMC simulation, we derive $\Omega_{m}=0.36_{-0.23}^{+0.31}$. For this test the other fitting parameters $\left(\alpha, \beta\right.$, and $\left.\mathcal{M}_{\lambda 1}\right)$ do not show any variation and remain similar within the uncertainties (variation only of $\sim 0.04$ on average for each parameter).

The shallow drop in the matter density distribution (Figure 2) and the relatively low intrinsic dispersion in the Hubble diagram obtained are encouraging for the derivation of cosmological parameters with reasonable uncertainties in the future. Indeed, with this method, we can correct the apparent 


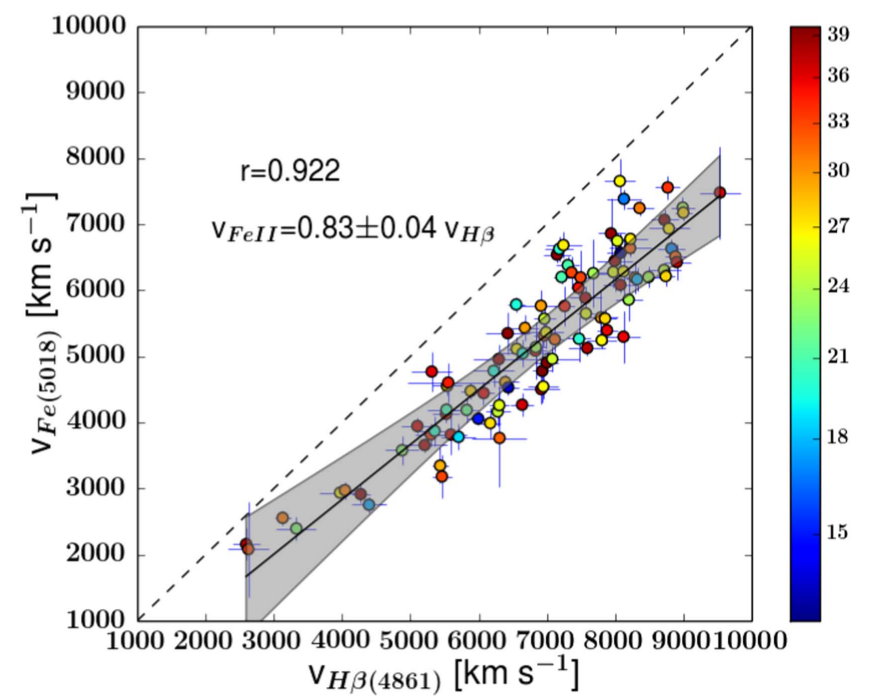

Figure 3. Velocities determined from the absorption minima of Fe II $\lambda 5018$ and $\mathrm{H} \beta \lambda 4861$. The dashed line represents $x=y$. In this figure, only the spectra of SNe II from the CSP-I sample at phases of 0-40 days after the explosion are plotted, i.e., 98 spectra. The shaded area is the $1 \sigma$ confidence interval using Scheffe's method. The color bar on the right side represents the different epochs from 0 to 40 days after the explosion.

magnitude using solely photometric input and thus add more $\mathrm{SNe}$ II in the Hubble diagram and at higher redshifts for which it is difficult to obtain spectrum with sufficient signal-to-noise ratio. This work demonstrates how the PCM can be extended to high redshift objects and will be an asset for the next generation of surveys. Even if SNe Ia offer more precise distances, our work suggests SNe II cosmology can be complementary, enabling even more precise measurements of the cosmological parameters.

\section{Standard Candle Method}

\subsection{Photospheric Expansion Velocities}

The SCM is the most used to standardize SNe II. This method is based on the correlation between the photospheric expansion velocities ( $\left.v_{\text {phot }}\right)$ and the intrinsic luminosity and so requires at least one spectrum, unlike the PCM. The precise measurement of the $v_{\text {phot }}$ is not possible because no spectral line is directly connected to this velocity. However, an estimate of $v_{\text {phot }}(5 \%-10 \%$ of accuracy; Dessart \& Hillier 2005) can be obtained through the minimum flux of the absorption component of the P-Cygni line profile of an optically thin line formed by pure scattering such as Fe II $\lambda 5018$ or Fe II $\lambda 5169$.

Measuring the Fe II absorption line for noisy or early $(\leqslant 20$ days) spectra can be very difficult, and therefore some authors attempt to use stronger features. Nugent et al. (2006) proposed to use the $\mathrm{H} \beta \lambda 4861$ absorption line, which is stronger than the weaker Fe II absorption line but also present in the early spectrum. The original correlation between the $\mathrm{H} \beta \lambda 4861$ and the Fe II velocities found by Nugent et al. (2006) was revisited recently by Poznanski et al. (2010) and Takáts \& Vinkó (2012). Using 28 spectra over 5 of 40 days after the explosion, Poznanski et al. (2010) found $v_{\mathrm{Fe}}$ II $=0.84 \pm 0.05 v_{\mathrm{H} \beta}$, a relation confirmed by Takáts \& Vinkó (2012), who found using the same range (between 5 of 40 days after the explosion) $v_{\mathrm{Fe} \text { II }}=0.823 \pm 0.015 v_{\mathrm{H} \beta}$. Using our spectral library at low redshift (CSP-I sample) and $\sim 100$ spectra between 0 and 40 days after the explosion, we derive a very consistent relation. As we can see in Figure 3 where we show the Fe II $\lambda 5018$ velocity versus the $\mathrm{H} \beta \quad \lambda 4861$ velocity, we obtain a strong correlation with a Pearson factor of 0.92 and a relation between both velocities defined as $v_{\mathrm{Fe} \text { II }}=0.83 \pm 0.04 v_{\mathrm{H} \beta}$ consistent with previous studies. Note that in this work, we use the Fe II $\lambda 5018$ line instead of Fe II $\lambda 5169$ because the latter can be blended by other elements such as the Fe triplet or Sc I

We use the $\mathrm{H} \beta \lambda 4861$ velocities to standardize the $\mathrm{SNe}$ II because the majority of the high redshift spectra (SDSS-II and SNLS samples) are noisy and taken at early phases where the Fe II absorption lines are not visible. Errors on the $\mathrm{H} \beta$ velocities were obtained by measuring many times the minimum of the absorption changing the continuum fit. Both quantities are listed in Table 1. To find the best epoch to use the SCM we need the velocities for different epochs. As proposed by Hamuy (2001) and used in all SNe II cosmology works (Nugent et al. 2006; Poznanski et al. 2009; D'Andrea et al. 2010; Olivares et al. 2010; Poznanski et al. 2010; Rodríguez et al. 2014; de Jaeger et al. 2015) we do an interpolation/ extrapolation using a power law of the form

$$
V(t)=A \times t^{\gamma},
$$

where $A$ and $\gamma$ are two free parameters obtained by leastsquares minimization for each individual $\mathrm{SN}$ and $t$ is the epoch since the explosion. To derive the velocity error following the work done by de Jaeger et al. (2015), a Monte Carlo simulation is performed, varying randomly each velocity measurement according to the observed velocity uncertainties over more than 2000 simulations. Following Poznanski et al. (2009), we add in quadrature to the velocity uncertainty of every SN II a value of $150 \mathrm{~km} \mathrm{~s}^{-1}$ to account for unknown host-galaxy peculiar velocities. For the SNe II with one spectrum the same power law is used but this time with a fixed $\gamma$, which is derived using only the CSP-I sample for which we have many spectra per SN and a better fit can be achieved. We find a median value of $\gamma=-0.407 \pm 0.173$. It is important to note that in the majority of other SN II cosmology works, the authors used the same power law for all of the $\mathrm{SNe}$, whereas in our work the $\gamma$ is different for all SNe II with more than two spectra. Additionally, in Section 6.4, we show the possibility of using a new relation between $A$ and $\gamma$ in order to derive the velocity when only one spectrum is acquired without assuming the same power-law exponent.

\subsection{Methodology}

To plot the Hubble diagram, as in Section 4, we run an MCMC calculation and minimize the negative log of the same likelihood function (Equation (6)) but now using another model where instead of $s_{2}$ we have now $\mathrm{H} \beta$ velocities:

$$
\begin{aligned}
m_{\lambda 1}^{\text {model }}= & \mathcal{M}_{\lambda 1}-\alpha \log _{10}\left(\frac{v_{\mathrm{H} \beta}}{\left\langle v_{\mathrm{H} \beta}>\mathrm{km} \mathrm{s}^{-1}\right.}\right) \\
& +\beta_{\lambda 1}\left(m_{\lambda 2}-m_{\lambda 3}\right)+5 \log _{10}\left(\mathcal{D}_{L}\left(z_{\mathrm{CMB}} \mid \Omega_{m}, \Omega_{\Lambda}\right)\right),
\end{aligned}
$$




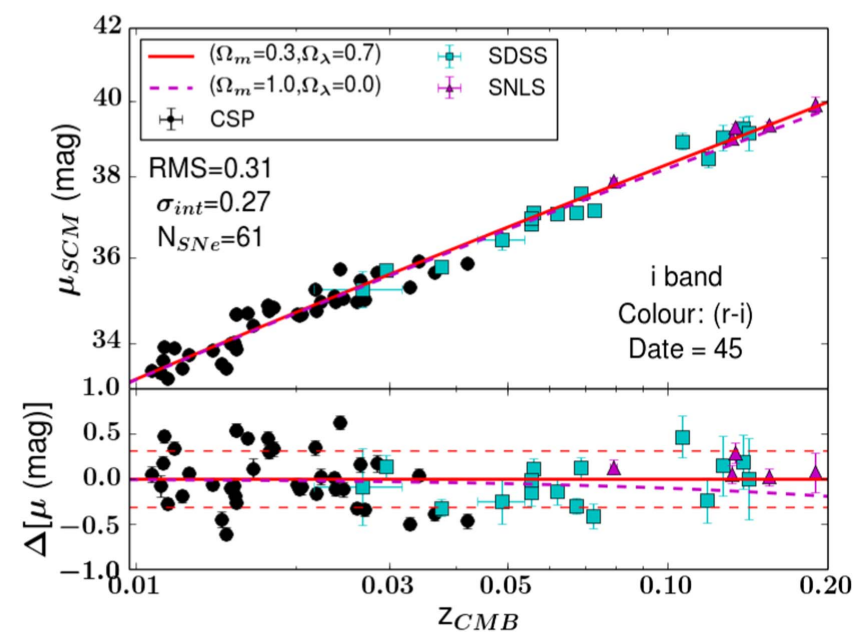

Figure 4. Hubble diagram for SNe II, using the SCM and all of the SNe II available at this epoch from the CSP-I, SDSS-II, and SNLS samples. Black dots represent the SNe II from the CSP-I whereas the cyan squares and magenta triangles are from the SDSS-II and the SNLS samples, respectively. The red line is the Hubble diagram for the $\Lambda$ CMB $\left(\Omega_{m}=0.3\right.$ and $\left.\Omega_{\Lambda}=0.7\right)$ and the magenta line is for an Einstein-de Sitter cosmological model $\left(\Omega_{m}=1.0\right.$ and $\Omega_{\Lambda}=0.0$ ). In both models, we assume a Hubble constant of $70 \mathrm{~km} \mathrm{~s}^{-1}$ $\mathrm{Mpc}^{-1}$. In the bottom panel, the residuals with respect to the $\Lambda \mathrm{CMB}$ are shown. We also present the number of SNe II available at this epoch $\left(N_{\mathrm{SNe}}\right)$, the epoch after the explosion (Date), the root mean square (rms), and the intrinsic dispersion $\left(\sigma_{\text {int }}\right)$.

where $\mathcal{D}_{L}\left(z \mid \Omega_{m}, \Omega_{\Lambda}\right), z_{\mathrm{CMB}}, \mathcal{M}_{\lambda 1}, \alpha$, and $\beta_{\lambda_{1}}$ are defined in the previous section and $\sigma_{\text {tot }}^{2}$ is defined as

$$
\begin{aligned}
\sigma_{\text {tot }}^{2}= & \sigma_{m_{\lambda 1}}^{2}+\left(\frac{\alpha}{\ln 10} \frac{\sigma_{v_{\mathrm{H} \beta}}}{v_{\mathrm{H} \beta}}\right)^{2}+\left(\beta \sigma_{\left(m_{\lambda 2}-m_{\lambda 3}\right)}\right)^{2} \\
& +\left(\sigma_{z} \frac{5(1+z)}{z(1+z / 2) \ln (10)}\right)^{2}+\sigma_{\mathrm{int} \cdot}^{2}
\end{aligned}
$$

Note that Equation (10) is the same one used by D'Andrea et al. (2010) and Poznanski et al. (2009) but they used the expansion velocity measured from the Fe II line instead of using the $\mathrm{H} \beta$ line as we do. As for the PCM, we center the velocity and color distributions, i.e., we divide the distribution by the mean velocity $\left(\left\langle v_{\mathrm{H} \beta}\right\rangle \sim 5900 \mathrm{~km} \mathrm{~s}^{-1}\right)$ and mean color $\left(\left\langle\left(m_{\lambda 2}-m_{\lambda 3}\right)\right\rangle \sim-0.02\right)$ of the whole sample respectively.

\subsection{Results}

\subsubsection{Fixed Cosmology}

The same color term as the PCM is used, and we plot the Hubble diagram for an epoch of 45 days in the rest-frame postexplosion. This epoch is the one with the smallest $\sigma_{\text {int }}$ and is consistent with 50 days in the rest-frame post-explosion used by other SN II cosmology works. Our sample at this specific epoch and combination is composed of $61 \mathrm{SNe}$ II. We find an intrinsic dispersion of $0.27 \mathrm{mag}$, i.e., $12 \%$ in distance errors. The use of the SCM allows us to reduce the intrinsic scatter from 0.55 mag (raw magnitudes) to $0.27 \mathrm{mag}$, i.e., an improvement of $13 \%$ in distance errors. We derive $\alpha=3.18_{-0.40}^{+0.41}, \beta=0.97_{-0.25}^{+0.26}$, and $\mathcal{M}_{\lambda 1}=-1.13_{-0.04}^{+0.04}$. Assuming a Hubble constant of $H_{0}=70 \mathrm{~km} \mathrm{~s}^{-1} \mathrm{Mpc}^{-1}$, an absolute magnitude $M_{i}=-16.91_{-0.04}^{+0.04}$ is obtained from $\mathcal{M}_{\lambda 1}$. The Hubble diagram and its associated Hubble residual are plotted in Figure 4. As we can see using this method, we are only able

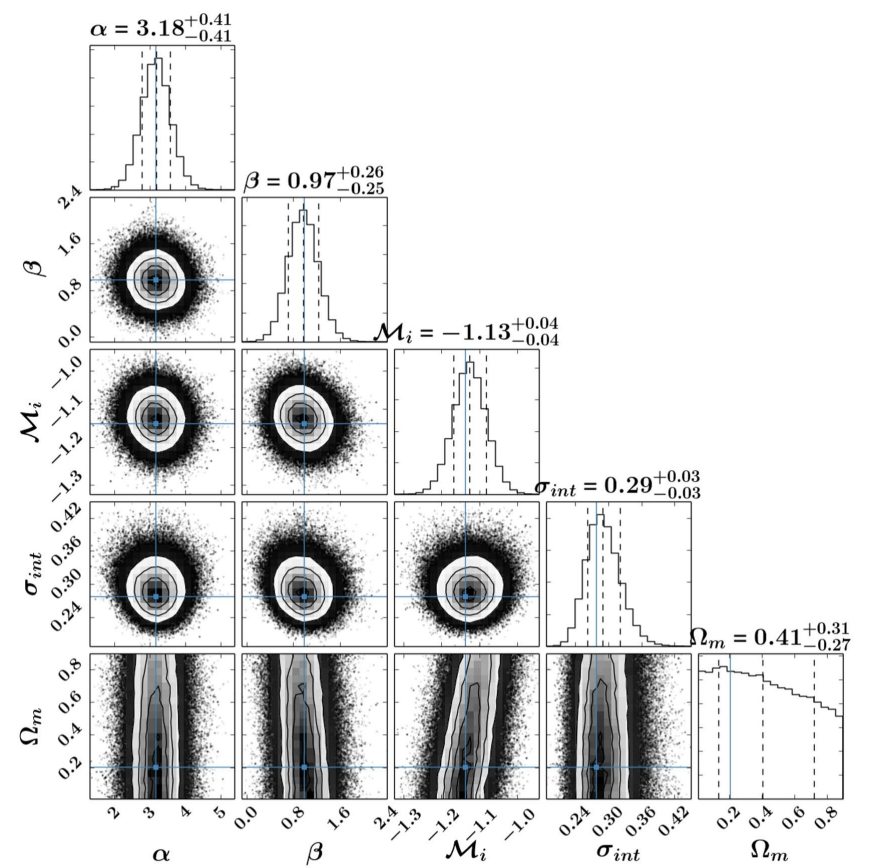

Figure 5. SCM: corner plot showing all the one- and two-dimensional projections. The blue lines are the values obtained using only one likelihood minimization. Contours are shown at $1 \sigma, 2 \sigma$, and $3 \sigma$. The five free parameters are plotted: $\alpha, \beta, \mathcal{M}_{\lambda 1}, \sigma_{\text {int }}$, and $\Omega_{m}$.

to reach redshift of around 0.2 where the distinction between cosmological models is very small.

We performed the same residual analysis between the data and the $\Lambda \mathrm{CDM}$ cosmological model and find the same conclusions: no autocorrelation, no outliers according to Chauvenet's criterion, and finally, we cannot reject the null hypothesis that the residuals come from a Gaussian distribution.

\subsection{2. $\Omega_{m}$ Derivation}

As done in Section 4.2.2, in this section we try to derive cosmological parameters. For the SCM the highest redshift used is too small to put constraints on the dark energy density and matter density. Despite this, the same MCMC calculation done in the Section 4.2.2 for a flat universe is performed. Figure 5 presents the same projections as Figure 2 but this time using the SCM. We find values consistent with that found with a fixed cosmology: $\alpha=3.18_{-0.41}^{+0.41}, \quad \beta=0.97_{-0.25}^{+0.26}$, and $\mathcal{M}_{\lambda 1}=-1.13_{-0.04}^{+0.04}$, and $\sigma_{\text {int }}=0.29_{-0.03}^{+0.03}$.

For the matter density we see a less pronounced drop than that obtained using the PCM. The value derived for the matter density is $\Omega_{m}=0.41_{-0.27}^{+0.31}$, which corresponds to $\Omega_{\lambda}=0.59_{-0.31}^{+0.27}$. In Figure 5 the blue lines represent the value derived using a simple likelihood minimization (without MCMC), e.g., for the density matter we obtain $\Omega_{m}=0.20 \pm 0.49$. The difference in the matter density error between the SCM and the PCM ( 0.49 versus 0.30$)$ is not due to the method (the intrinsic dispersion is better for the SCM) but is due to the redshift range and the number of SNe II. We clearly require higher redshift SNe II $(z \geqslant 0.3)$ to derive cosmological parameters and obtain better constraints on the matter density. 

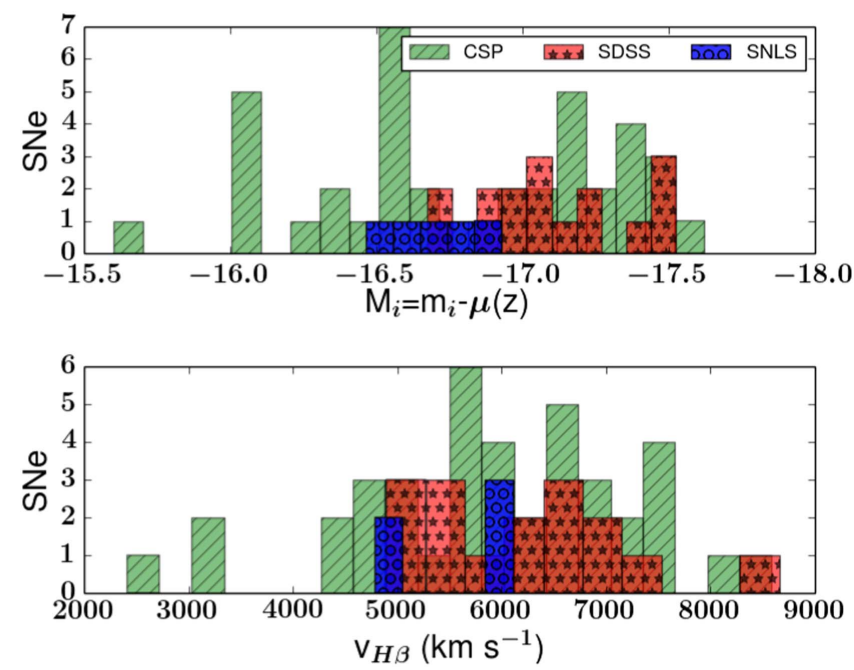

Figure 6. Comparison of the CSP-I, SDSS-II, and SNLS samples. Top panel represents the absolute magnitude without any calibration (not corrected for velocity or dust) and assuming a Hubble constant of $70 \mathrm{~km} \mathrm{~s}^{-1} \mathrm{Mpc}^{-1}$, $\Omega_{m}=0.3$, and $\Omega_{\Lambda}=0.7$. The bottom panel shows the distribution of $\mathrm{H} \beta$ velocity. In both plots, the green color represents the CSP-I sample, the red the SDSS-II sample, and the blue the SNLS sample.

\section{Discussion}

\subsection{Sample Comparison}

In this part, we will compare the three samples used for this work and with the SCM: CSP-I, SDSS-II, and SNLS. In Figure 6 (top), we compare the absolute magnitude uncorrected for velocity or host extinction and assuming a standard cosmology $\left(\Omega_{m}=0.3\right.$, and $\left.\Omega_{\Lambda}=0.7\right)$. Even if the number of SNe II used is very different (40 for CSP-I, 16 for SDSS-II, and 5 for SNLS), the luminosity distribution appears different. The CSP-I sample has absolute magnitudes over a range of 2 magnitudes, which is expected for SNe II. For the SDSS-II sample, as found by D'Andrea et al. (2010), it spreads only around a small range of absolute magnitude ( $0.7 \mathrm{mag})$. The authors explained the lack of any $\operatorname{dim}$ SNe II above $z=0.10$ by the Malmquist bias. At low redshift, we do not have intrinsically dimmer SNe II in the SDSS-II sample, due to the fact that dimmer candidates were not followed spectroscopically. For the SNLS sample, the statistic is too low to derive conclusions. The same result is found by analyzing the distribution of $\mathrm{H} \beta$ velocities. The CSP-I sample spreads a large range of velocities $\left(2500-8500 \mathrm{~km} \mathrm{~s}^{-1}\right)$ while the SDSS-II sample has in general high velocities $\left(5000-8000 \mathrm{~km} \mathrm{~s}^{-1}\right)$. The lack of low velocities for the SDSS-II sample could be explained by the bias toward more luminous $\mathrm{SNe}$ II in the SDSS-II sample. This bias could explain the higher dispersion in the Hubble diagram for the low-redshift SNe II. SNe II from the CSP-I sample spread over a larger range in observed properties than the SDSS-II sample, which are biased toward more luminous events. In the future, with larger data sets and simulations (see Section 6.7 for the Malmquist bias), we will be able to better characterize systematic biases.

\subsection{PCM versus SCM}

Using a larger data sample and higher redshift SNe II than de Jaeger et al. (2015) ( 40 SNe II up to $z \sim 0.04$ with $\sigma_{\text {int }}=0.41 \mathrm{mag}$ ), we obtain an intrinsic dispersion of 0.35 mag with the PCM and 0.27 mag with the SCM. The SCM is a

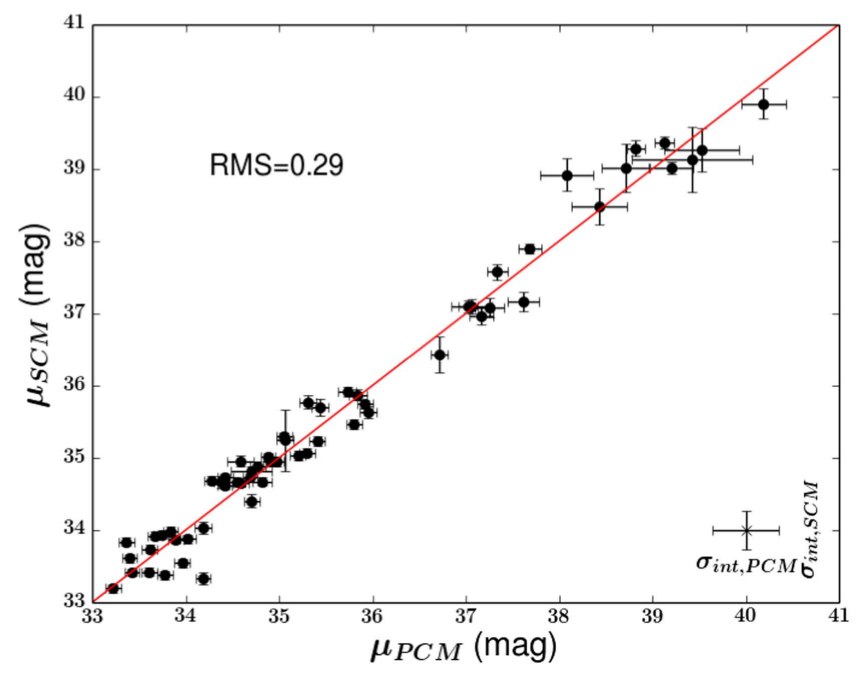

Figure 7. Comparison of distance modulus obtained using the PCM (x-axis) and the SCM (y-axis). The red line shows $x=y$. Note that the error bars do not include the intrinsic scatter $\left(\sigma_{\text {int }}\right)$ of each method, which are represented by the cross on the bottom right of the figure.

better method to standardize the SNe II in terms of intrinsic dispersion, but the difference between both methods is only $0.08 \mathrm{mag}$, i.e., $3 \%$ in distances. In contrast to the SCM, with the PCM we are able to use more SNe II (73 versus 61), and it can be extended to higher redshifts $(\sim 0.5$ versus $\sim 0.2)$. The next generation of telescopes will observe many thousands of SNe II and the PCM will be very useful in deriving cosmological parameters. In Figure 7 we present the distance modulus obtained using the PCM and the SCM. For these two methods, we have $59 \mathrm{SNe}$ II in common. As we can see, the values derived are very consistent using both methods, with an rms of $0.29 \mathrm{mag}$. All the distance moduli calculated for both methods are listed in Table 1.

\subsection{SCM versus Other Works}

The scatter found in this work is very consistent with those found by previous studies (Nugent et al. 2006; Poznanski et al. 2009; D'Andrea et al. 2010; Olivares et al. 2010; Poznanski et al. 2010; Rodríguez et al. 2014; de Jaeger et al. 2015). For example, following a similar methodology (same likelihood), D'Andrea et al. (2010), using 15 SNe II from SDSS-II with 34 low-redshift SNe II from Poznanski et al. (2009), found an intrinsic dispersion of $0.29 \mathrm{mag}$. They also derived consistent free parameters $\alpha=4.0 \pm 0.7$, $\beta=0.8 \pm 0.3$ but a different absolute magnitude $M_{I}=-17.52 \pm 0.08$ mag. This is largely because they assumed an intrinsic color of 0.53 mag to correct their magnitudes for extinction.

Poznanski et al. (2009) also found similar dispersion, i.e., $0.38 \mathrm{mag}$, using $40 \mathrm{SNe}$ II ("full") or $0.22 \mathrm{mag}$ after removing six outliers ("culled"). In Table 3 we present the values of $\alpha, \beta$, $M_{I}$, and $\sigma_{\text {int }}$ from different works (values taken from Table 4 of D'Andrea et al. 2010) and using our different samples (CSP-I, SDSS-II, SNLS). As we can see from this table, even if the free parameters are consistent with our work, small differences are present. For example, the discrepancy in the value of $\alpha$ could be explained by the method used. In this paper, we use the $\mathrm{H} \beta$ velocity while the other two studies used the iron line. We also calculate a power law for the majority of the SNe II for the velocity while both authors assumed a unique power law for all 
Table 3

PCM-fit Parameters

\begin{tabular}{|c|c|c|c|c|c|}
\hline Data Set & $\alpha$ & $\beta$ & $M_{i}$ & $\sigma_{\text {int }}$ & $\mathrm{SNe}$ \\
\hline Poznanski et al. (2009) "full" & $4.4 \pm 0.7$ & $0.6_{-0.4}^{+0.3}$ & $-17.42 \pm 0.10$ & 0.35 & 40 \\
\hline Poznanski et al. (2009) "culled" & $4.2 \pm 0.6$ & $0.8_{-0.3}^{+0.3}$ & $-17.38 \pm 0.08$ & 0.20 & 34 \\
\hline SDSS-II from D'Andrea et al. (2010) & $1.8_{-1.0}^{+0.9}$ & $0.1 \pm 0.5$ & $-17.67_{-1.0}^{+0.11}$ & 0.16 & 15 \\
\hline D'Andrea et al. (2010) + Poznanski et al. (2009)“culled" & $4.0 \pm 0.7$ & $0.8_{-0.3}^{+0.3}$ & $-17.52 \pm 0.08$ & 0.29 & 49 \\
\hline CSP-I & $3.04_{-0.47}^{+0.48}$ & $1.54_{-0.37}^{+0.38}$ & $-16.85_{-0.05}^{+0.05}$ & $0.31_{-0.03}^{+0.04}$ & 40 \\
\hline CSP-I+SDSS-II & $3.16_{-0.42}^{+0.42}$ & $1.01_{-0.27}^{+0.28}$ & $-16.92_{-0.05}^{+0.05}$ & $0.30_{-0.03}^{+0.03}$ & 56 \\
\hline CSP- I+SNLS & $3.05_{-0.44}^{+0.45}$ & $1.41_{-0.35}^{+0.35}$ & $-16.84_{-0.05}^{+0.05}$ & $0.30_{-0.03}^{+0.04}$ & 45 \\
\hline SDSS-II+SNLS & $3.55_{-0.77}^{+0.82}$ & $0.39_{-0.24}^{+0.25}$ & $-17.02_{-0.05}^{+0.05}$ & $0.18_{-0.04}^{+0.05}$ & 21 \\
\hline CSP-I+SDSS-II+SNLS & $3.18_{-0.41}^{+0.41}$ & $0.97_{-0.25}^{+0.26}$ & $-16.91_{-0.04}^{+0.04}$ & $0.29_{-0.03}^{+0.03}$ & 61 \\
\hline
\end{tabular}

Note. Best-fit values and the associated errors for each parameter for different samples using the SCM.

SNe. Thus, these differences could have an impact on the $\alpha$ value. For $\mathcal{M}_{\lambda 1}$ as stated previously, we do not correct the color for intrinsic color, which could affect the value derived for $\mathcal{M}_{\lambda 1}$. Additionally, the discrepancies in $\beta$ and $\mathcal{M}_{\lambda 1}$ could arise from differences in the filters used. They used the Bessel filters $R$ and $I$ while we used the CSP-I filters $r$ and $i$.

Note also that the differences in methodology are not the only cause affecting the SCM fit parameters. D'Andrea et al. (2010) explained that these effects could arise from selection effects as described in Section 6.1

We can also compare our distance moduli with those derived by Poznanski et al. (2009). The Poznanski et al. (2009) sample and our sample share two SNe II: 04D1pj and 04D4fu. Poznanski et al. (2009) derived a distance modulus of $39.28 \pm 0.11$ and $38.85 \pm 0.11$ while we obtain $39.367 \pm 0.084$ and $39.018 \pm 0.087$ for $04 \mathrm{D} 1 \mathrm{pj}$ and 04D4fu, respectively. These two values are very consistent.

\section{4. $H \beta$ Velocity: $A$ and $\gamma$ Correlation}

Pejcha \& Prieto (2015) found a correlation between the two free parameters $(A$ and $\gamma$ ) used in the expansion velocity formula described in Equation (9). They found that velocity decays faster in SNe II with initially higher velocity. Using all of the SNe II from the CSP-I sample with more than three spectra (46 SNe II), we present in Figure 8 the plot of the power-law exponent $(\gamma)$ versus the initial velocity $(A)$. As we can see, our observational data confirm the result found by Pejcha \& Prieto (2015): SNe II with high initial velocity decay faster. Additionally, we remark that the shape of both relations (from Pejcha \& Prieto 2015 and ours) is very consistent. They found a bi-modal correlation, but with $\gamma$ lower because in their model a constant velocity offset is added. This typically makes $\gamma$ more negative. Note that we find similar correlation factors, -0.82 and -0.86 for their work and our study, respectively. The relation between these quantities is very important to derive the expansion velocity for the SNe II with only one spectrum. In the literature, the majority of the studies assumed the same power-law exponent for all $\mathrm{SNe}$ II or assumed a median value for the SNe II with only one spectrum (as done in Section 5.1). However, thanks to this relation, we can derive the $\mathrm{H}_{\beta}$ velocity with more accuracy. In Figure 8 we show four different fits: a power law (black), a linear fit (blue), an inverse fit (green), and a bi-modal fit (cyan). The best reduced chisquare and dispersion are obtained using the bi-modal fit (16 and 0.08 , respectively). If the $\mathrm{H}_{\beta}$ velocities for the $\mathrm{SNe}$ II with only one spectrum are derived using the two-line fit, i.e.,

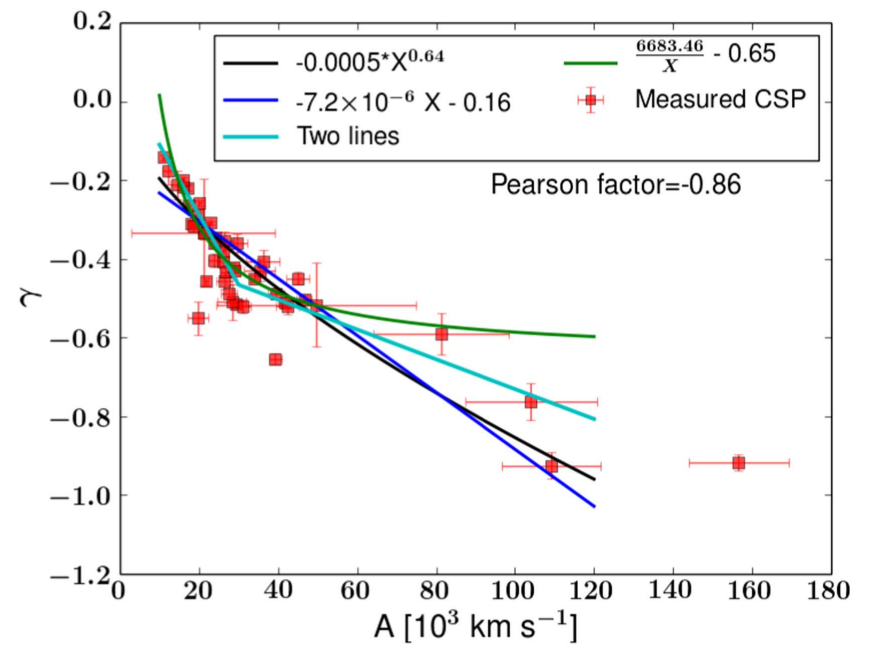

Figure 8. $\gamma$ vs. $A$ using the CSP-I sample. The red squares represent the CSP-I sample; the black line is a power-law fit, the blue line is a linear fit, the green line is an inverse fit, and in cyan a two-line fit.

$\gamma=-1.71 \times 10^{-5} A+5.25 \times 10^{-2}$ for $A \leqslant 30500$ or $\gamma=-3.82 \times 10^{-6} A-0.35$ for $A \geqslant 30500$, we are able to derive a Hubble diagram with an equivalent dispersion $\left(\sigma_{\mathrm{int}} \sim 0.28 \mathrm{mag}\right)$ to that derived in Section 5.3.2.

\subsection{Sensitivity to Progenitor Metallicity?}

Using theoretical models, Kasen \& Woosley (2009) suggested that progenitors with different metallicities $\left(Z=0.1-1 Z_{\odot}\right)$ could introduce some systematic errors $(0.1 \mathrm{mag})$ in the photospheric expansion velocity-intrinsic luminosity relation. From an observational point of view, Anderson et al. (2016) and Taddia et al. (2016), using SNe II from the CSP-I and the intermediate Palomar Transient Factory (iPTF), respectively, showed that the equivalent width of the $\mathrm{Fe}$ II $\left(\mathrm{EW}_{\mathrm{Fe}}\right)$ and the absolute magnitude at maximum peak are correlated in the sense that $\mathrm{SNe}$ II with smaller $\mathrm{EW}_{\mathrm{Fe}}$ tend to be brighter.

In this part, we aim to study this using only the CSP-I sample, which is the only available sample where metal line measurements are possible. We linearly interpolate the equivalent width to 45 days post-explosion and for this specific epoch, and end up with a sample of $25 \mathrm{SNe}$ II. Note that an MC simulation is performed by randomly varying each $\mathrm{EW}_{\mathrm{Fe}}$ measurement according to their uncertainties and linearly interpolate at epoch 45 days post-explosion. We then take as 


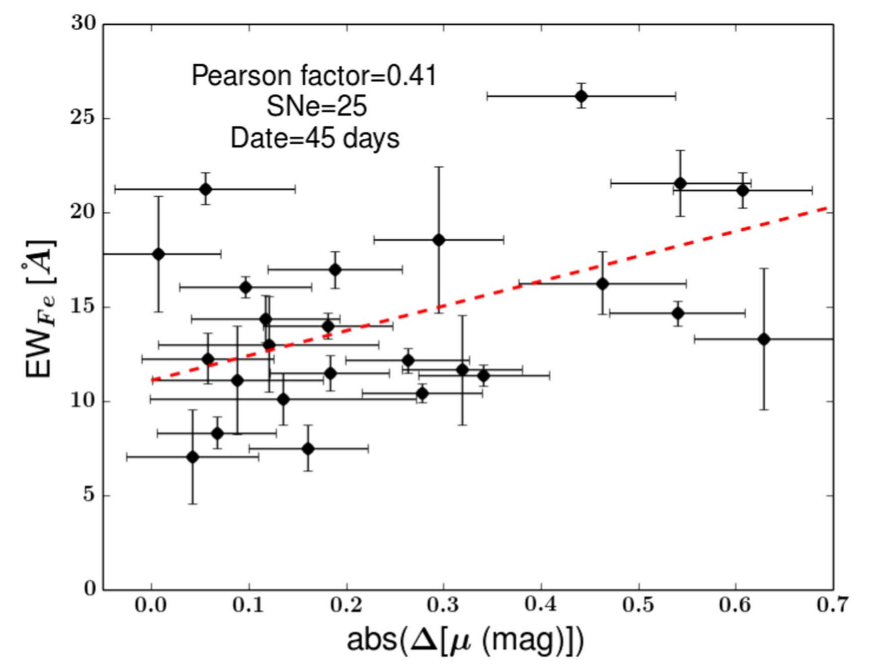

Figure 9. Absolute Hubble residual using the SCM and the $\Lambda$ CDM cosmological model vs. the equivalent width of the Fe II $\lambda 5018$ absorption line. The red dashed line represents the linear regression, taking into account errors in both variables using the Python port of B. Kelly's LINMIX ERR IDL package (Kelly 2007, https://github.com/jmeyers314/linmix).

the final $\mathrm{EW}_{\mathrm{Fe}}$ the median while the error is the standard deviation of these 2000 fits.

Figure 9 shows $\mathrm{EW}_{\mathrm{Fe}}$ versus the absolute Hubble diagram residual to the $\Lambda \mathrm{CDM}$ model $\left(\Omega_{m}=0.3\right.$ and $\left.\Omega_{\Lambda}=0.7\right)$ and using the SCM. We find a trend between the $\mathrm{EW}_{\mathrm{Fe}}$ and the absolute residual, i.e., SNe II with smaller $\mathrm{EW}_{\mathrm{Fe}}$ have less dispersion. The Pearson factor of 0.41 confirmed this tiny relation. This figure could reflect the existence of one category of SNe II more standardizable than others, i.e., SNe II with small $\mathrm{EW}_{\mathrm{Fe}}(<10 \AA)$ seem to be better standard candles than the others. It will be very interesting to construct a Hubble diagram using only $\mathrm{SNe}$ II with small $\mathrm{EW}_{\mathrm{Fe}}$, but unfortunately a sufficient number of $\mathrm{SNe}$ II are unavailable. If the Hubble diagram residual is taken instead of the absolute of the Hubble diagram residual, no correlation is found with the $\mathrm{EW}_{\mathrm{Fe}}$. Note that in our Hubble diagram (Figure 4) the higher redshift SNe II (SDSS-II, SNLS) seem to have less intrinsic dispersion than the low-redshift sample. This could be also explained by the fact that higher redshift $\mathrm{SNe}$ II have a smaller range in luminosity (Section 6.1), and thus a smaller range in $\mathrm{EW}_{\mathrm{Fe}}$, which could imply less scatter.

If we use the equivalent width of the Fe II $\lambda 5018$ absorption line as a proxy for metallicity (Dessart et al. 2014), and if the Hubble diagram residual is only coming from the metallicity, we can conclude, as Kasen \& Woosley (2009), that differences in metallicity introduce some scatter in the Hubble diagram. All the figures and discussions regarding metallicity will be left to a future publication (C. P. Gutíerrez et al. 2016, in preparation).

\subsection{Combined SNe Ia and SNe II Hubble Diagram}

In this section, we combine our SNe II sample with a complete SNe Ia sample (740 SNe Ia) from Betoule et al. (2014). In Figure 10, we show the combined Hubble diagram where both samples are fitted separately, i.e., using the distance modulus derived with the PCM for the SNe II and the distance modulus using the fitting parameters from Betoule et al. (2014) for the SNe Ia sample. Then, we try to fit simultaneously both samples following the work done by Scovacricchi et al. (2016) where they combined 100 simulated superluminous SNe from

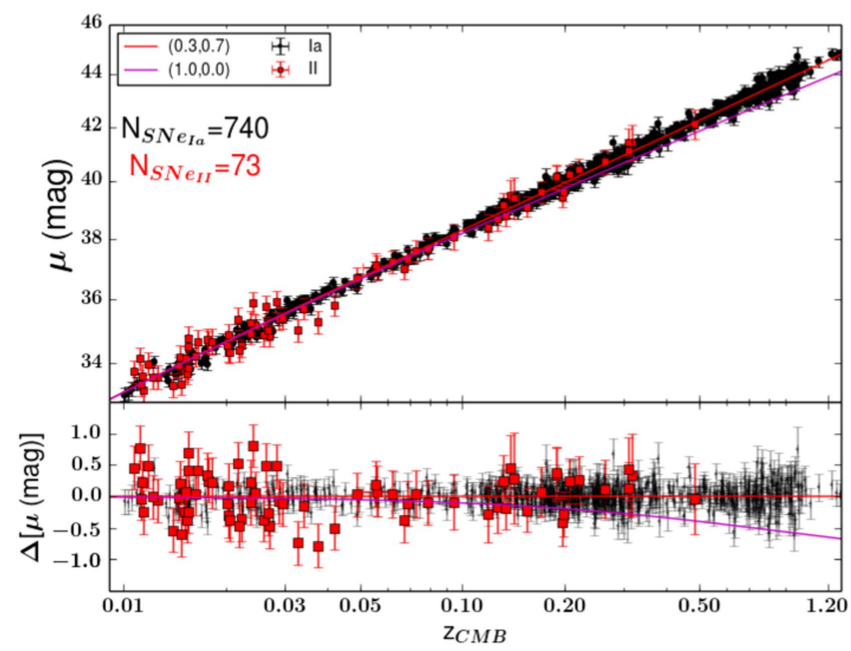

Figure 10. Combined Hubble diagram using SNe Ia from Betoule et al. (2014) and SNe II. The red squares are for the SNe II (using the PCM) and the black dots are for the SNe Ia. Note that distance modulus errors for both methods include the intrinsic dispersion. The red line shows the Hubble diagram for the $\Lambda$ CMB $\left(\Omega_{m}=0.3\right.$ and $\left.\Omega_{\Lambda}=0.7\right)$ and the magenta line shows an Einstein-de Sitter cosmological model $\left(\Omega_{m}=1.0\right.$ and $\left.\Omega_{\Lambda}=0.0\right)$.

SUDSS and 3800 SNe Ia from DES. We minimize the likelihood corresponding to the product of two likelihoods $\mathcal{L}=\mathcal{L}_{\mathrm{Ia}} * \mathcal{L}_{\mathrm{II}}$. We thus have nine free parameters: $\alpha, \beta, \mathcal{M}_{\lambda 1}$, and $\sigma_{\text {int }}$ for the two likelihoods plus the same $\Omega_{m}$. Note that for the SNe Ia sample we use the same likelihood used in Equation (6) but, instead of the $s_{2}$ value or the $\mathrm{H} \beta$ velocity, we use the stretch parameter. To estimate the effect of combining the two samples, we look at the value derived for $\Omega_{m}$ and especially its uncertainty. Using the PCM or the SCM, the precision derived for the matter density with the combined samples is not better than the one obtained using only the SNe Ia sample. This can be easily explained by three different factors: the redshift range (SNe Ia up to 1.2), the size of the sample, and the fact that $\mathrm{SNe}$ Ia are more standardizable $\left(\sigma_{\text {int }} \sim 0.10-0.15 \mathrm{mag}\right)$.

To compare the difference in precision achieved with the SNe Ia and SNe II, we restrict the SNe Ia sample to the same $\mathrm{SNe}$ II redshift range, i.e., $z \leqslant 0.5$. Doing a Monte Carlo simulation (hundred iterations), $73 \mathrm{SNe}$ Ia (equivalent to the SNe II sample size) are randomly selected. A median uncertainty in the matter density of 0.1 is derived, which compares to the $\sim 0.3$ using only SNe II. Otherwise, we can count how many SNe Ia are necessary to reach a precision of $\sim 0.3$ in the density matter compared to the $73 \mathrm{SNe}$ II needed. We find that $22 \mathrm{SNe}$ Ia or $13 \mathrm{SNe}$ Ia are required using the $\mathrm{PCM}$ and the SCM, respectively. This corresponds to $\sim 30 \%$ $( \pm 8 \%)$ or $\sim 20 \%( \pm 7 \%)$ of the SNe II sample size for the PCM and the SCM, respectively. Individually, even if the SNe Ia are better standard candles than the SNe II, SNe II cosmology can provide an independent measurement of the cosmological parameters. Or, with growing samples in the future, they may be used, as shown here, in combination with SNe Ia. As stated earlier, SN II progenitors are better understood than those of $\mathrm{SNe}$ Ia (given their direct detection in pre-explosion images; e.g., Smartt et al. 2009), which may allow us to further reduce the intrinsic dispersion, possibly reaching the same dispersion offered by SNe Ia. 


\subsection{Malmquist Bias}

Using a basic simulation, we investigate the Malmquist bias, which leads to the observation of preferentially brighter objects in a magnitude limited survey. This bias implies a mean observed magnitude brighter than the intrinsic mean, biasing distance measurements and therefore overestimating the matter density. This definition is only valid if the magnitudes have a Gaussian distribution, which is the case for the $\mathrm{SNe}$ (see Anderson et al. 2014 for magnitude distributions at different epochs.)

To derive an approximate Malmquist bias, we calculate the difference in magnitudes after applying the PCM ( $s_{2}$ and color corrections) of fake input $\mathrm{SNe}$ II distance moduli and output SNe II distance moduli, i.e., the SNe II that have passed the limiting magnitude cut of the survey. The $(g, r, i, z)$ limiting magnitudes assumed are $(25.5,25.0,24.8,23.9)$ and (22.2, 22.2, 21.3, 20.5) for SNLS and SDSS-II, respectively.

To generate fake apparent magnitudes, we adopt our lowredshift sample distribution (CSP-I), i.e., the absolute $V$-band magnitudes at the end of the plateau $\left(M_{\text {end }}\right)$ between -15.0 and -17.0 (Anderson et al. 2014). Using MC simulations and the correlation matrix between $M_{\text {end }}$, the $s_{2}$, the plateau duration $(\mathrm{Pd})$, and the colors $(B-V)$, we generate 10,000 mock SNe II that follow the nearby distribution. Due to the fact that it is a simplistic simulation, the effects of observing conditions (such as seeing, sky transparency, etc.) are not modeled. However, we do take into account the same magnitude errors of the actual observed SNe of the CSP-I survey and inject them into the fake SNe. Then, we randomly select 2000 SNe II (i.e., $\sim 2000$ sets of $\left.M_{\text {end }}, s_{2}, \mathrm{Pd},(B-V)\right)$ from the MC simulation, and for each SN II we use $\sim 2000$ random redshifts between 0.03 and 0.80 . From this, we can derive an apparent magnitude (i.e., $\sim 4,000,000$ magnitudes) assuming a $\Lambda \mathrm{CDM}$ model and applying an inverse K-correction (similar to Section 3.2). Then, we compare the apparent magnitude (at the end of the plateau) with the limiting magnitude, which depends on the redshift. For a redshift $\leqslant 0.3$, an absolute magnitude in the $V$ band corresponds to an apparent magnitude in the $r$ band; from $0.3<z<0.56$ to the $i$ band; and finally from $0.56<z<0.80$ to the $z$ band. All of the SNe II that pass this cut form our output sample. From the input and output SNe II, we can derive the modulus distances after correcting the magnitude using $\alpha=0.37 \pm 0.10$ and $\beta=1.20 \pm 0.35$. The $\alpha$ and $\beta$ values were derived using only the CSP-I sample and applying the PCM as achieved in de Jaeger et al. (2015). The final Malmquist bias is taken as the mean value of the modulus distance difference of the input and output samples for a redshift bin of 0.02 . Then, we interpolate linearly the Malmquist bias over all of the bins in order to apply this bias to each SN of the SDSS and SNLS samples. The errors for each bin are taken as the standard error of the mean. For clarity, the standard deviation ( $\sim 0.5 \mathrm{mag})$ is not shown in this figure.

Roughly, for the SNLS survey, we derive a mean Malmquist bias of $\sim-0.02 \mathrm{mag}$ for a redshift range 0.02-0.3. After this range, the mean Malmquist bias decreases up to $\sim-0.16 \mathrm{mag}$ for a redshift between 0.3 and 0.4 , and finally to $\sim-0.32$ mag for a redshift between 0.4 and 0.5 . The Malmquist bias decreasing after $z=0.22$ can be modeled by a straight line of equation: $\mathrm{MB}_{\mathrm{SNLS}}=-1.21( \pm 0.10) \times z+0.25( \pm 0.03)$.

Similarly, for the SDSS survey, we derive a mean Malmquist bias of $\sim-0.08 \mathrm{mag}$ for the redshift range $0.02-0.1$ and $\sim-0.31 \mathrm{mag}$ for the redshift range $0.1-0.15$. As for SNLS, we

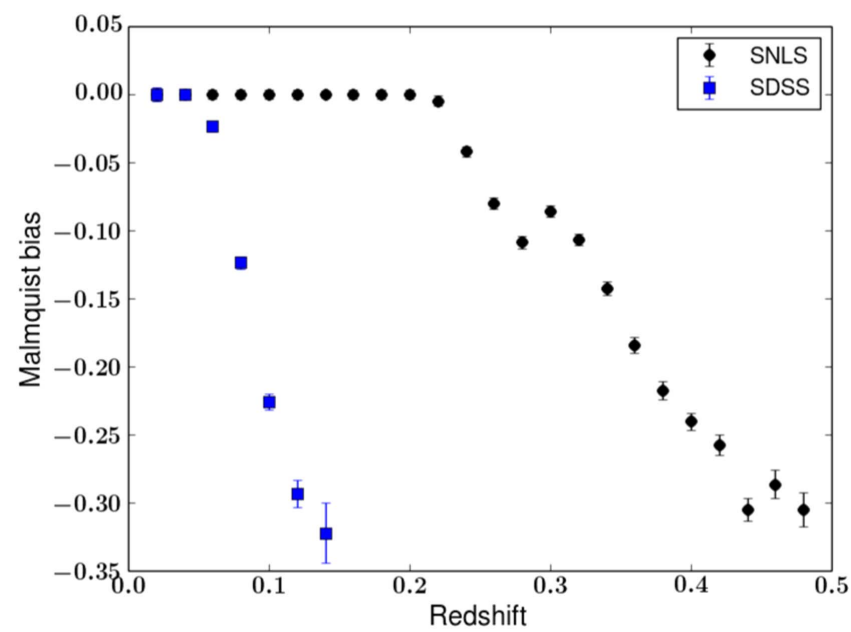

Figure 11. Squares represent the simulated Malmquist bias vs. redshift for the SNLS and SDSS-II survey in black and blue, respectively.

fit the decreasing Malmquist bias after $z=0.06$ with a straight line of equation $\mathrm{MB}_{S D S S}=-4.61( \pm 0.55) \times z+0.23( \pm 0.05)$. In Figure 11, we present this approximate Malmquist bias versus redshift. The high values derived for all surveys are a warning for SNe II cosmology. Deriving strong constraints for the cosmological parameters requires measurements extending far back in time when the Malmquist bias is important. Thus, it will be difficult to reach the same level of precision as that obtained with the SNe Ia for which the Malmquist bias (Perrett et al. 2010) and the intrinsic dispersion are much smaller. In the future, it is crucial to obtain a good estimation of this bias with a full simulation as achieved by Perrett et al. (2010).

Even if our method is an approximation, we apply the Malmquist bias to each SN II in our Hubble diagram. For this, to each SN II-corrected apparent magnitude, the value of the Malmquist bias is added at the SN II redshift. Then, we derive the matter density and compare it with the value obtained in Section 4.2.2. The matter density distribution shape is very similar to that derived in Figure 2 and the value obtained, $\Omega_{m}=0.35_{-0.22}^{+0.30}$, is also very consistent. We again caution the reader that even if the Malmquist bias does not seem to affect our cosmology it should be calculated with more accuracy.

\section{Conclusions}

Using three samples, CSP-I, SDSS-II, and SNLS, we construct the two largest SNe II Hubble diagrams (73 SNe II in this work versus $49 \mathrm{SNe}$ II in the literature), extending successfully the Photometric Color Method developed in de Jaeger et al. (2015) to higher redshifts (up to 0.5). We also compare this method with the SCM. In summary,

1. Using the PCM we find an intrinsic dispersion of 0.35 mag (73 SNe II with a redshift up to $~ 0.5$ ) using the $i$ band, while using the SCM we obtained a dispersion of $0.27 \mathrm{mag}$ (61 SNe II with a redshift up to $\sim 0.2$ ).

2. The Hubble diagram derived from the CSP-I+SDSS-II + SNLS samples using the SCM yields a dispersion similar to those found in the literature and emphasises the potential of SCM in cosmology.

3. We find a relation between the power-law exponent $(\gamma)$ and the initial velocity $(A)$, which is very useful to derive $\mathrm{H}_{\beta}$ velocities at any epoch with only one $\mathrm{SNe}$ II spectrum. 
4. We derived cosmological parameters $\left(\Omega_{m}\right)$ consistent with the $\Lambda$ CDM. Using the PCM we found $\Omega_{m}=0.32_{-0.21}^{+0.30}$, and with the SCM $\Omega_{m}=0.41_{-0.27}^{+0.31}$. These results are consistent with the existence of dark energy at $2 \sigma$.

5. The distance moduli derived using the PCM and the SCM are very consistent with a dispersion of $0.29 \mathrm{mag}$.

6. Using a simple simulation, we point out the high values for the SNe II Malmquist bias, which could be problematic to achieve in the future, and cosmological constraint uncertainties similar those obtained with the SNe Ia.

While SNe II currently display larger scatter in their use as distance indicators compared to $\mathrm{SNe}$ Ia, in this work we have shown that SNe II can be used as viable independent cosmological probes. Indeed, with future large surveys predicted to significantly extend the number of $\mathrm{SNe}$ II at higher redshift, these objects promise to provide a valuable sanity check to results obtained from other methods.

Support for T.D., S.G., M.H., H.K., and C.G. is provided by the Ministry of Economy, Development, and Tourism's Millennium Science Initiative through grant IC120009, awarded to The Millennium Institute of Astrophysics, MAS. S.G. acknowledges support from Basal Project PFB03. H.K. also acknowledges support by CONICYT through FONDECYT grants 3140566 and 3140563, respectively. L.G. was supported in part by the US National Science Foundation under Grant AST1311862. M.D.S gratefully acknowledges the generous support provided by the Danish Agency for Science and Technology and Innovation realized through a Sapere Aude Level 2 grant. M.S. acknowledges support from EU/FP7-ERC grant 615929. The work of CSP-I has been supported by the National Science Foundation under grants AST0306969, AST0607438, and AST1008343. This research has made use of the NASA/IPAC Extragalactic Database (NED), which is operated by the Jet Propulsion Laboratory, California Institute of Technology, under contract with the National Aeronautics and Space Administration and of data provided by the Central Bureau for Astronomical Telegrams. This work is based in part on data produced at the Canadian Astronomy Data Centre as part of the CFHT Legacy Survey, a collaborative project of the National Research Council of Canada and the French Centre National de la Recherche Scientifique. This work is also based on observations obtained at the Gemini Observatory, which is operated by the Association of Universities for Research in Astronomy, Inc., under a cooperative agreement with the NSF on behalf of the Gemini partnership: the National Science Foundation (United States), the STFC (United Kingdom), the National Research Council (Canada), CONICYT (Chile), the Australian Research Council (Australia), $\mathrm{CNPq}$ (Brazil) and CONICET (Argentina). This research used observations from Gemini program numbers: GN-2005A-Q-11, GN2005B-Q-7, GN-2006A-Q-7, GS-2005A-Q-11 and GS-2005BQ-6, and GS-2008B-Q-56.

\section{References}

Anderson, J. P., González-Gaitán, S., Hamuy, M., et al. 2014, ApJ, 786, 67 Anderson, J. P., Gutierrez, C. P., Dessart, L., et al. 2016, A\&A, 589, A110 Arcavi, I., Gal-Yam, A., Cenko, S. B., et al. 2012, ApJL, 756, L30

Astier, P., Guy, J., Regnault, N., et al. 2006, A\&A, 447, 31

Balland, C., Baumont, S., Basa, S., et al. 2009, A\&A, 507, 85
Baron, E., Nugent, P. E., Branch, D., \& Hauschildt, P. H. 2004, ApJL, 616, L91

Baumont, S., Balland, C., Astier, P., et al. 2008, A\&A, 491, 567

Bazin, G., Palanque-Delabrouille, N., Rich, J., et al. 2009, A\&A, 499, 653

Betoule, M., Kessler, R., Guy, J., et al. 2014, A\&A, 568, A22

Boulade, O., Charlot, X., Abbon, P., et al. 2003, Proc. SPIE, 4841, 72

Bronder, T. J., Hook, I. M., Astier, P., et al. 2008, A\&A, 477, 717

Cardelli, J. A., Clayton, G. C., \& Mathis, J. S. 1989, ApJ, 345, 245

Chauvenet, W. 1863, A Manual of Spherical and Practical Astronomy V. II (5th ed.; New York, NY: Dover)

Conley, A., Guy, J., Sullivan, M., et al. 2011, ApJS, 192, 1

Contreras, C., Hamuy, M., Phillips, M. M., et al. 2010, AJ, 139, 519

D’Andrea, C. B., Sako, M., Dilday, B., et al. 2010, ApJ, 708, 661

de Jaeger, T., González-Gaitán, S., Anderson, J. P., et al. 2015, ApJ, 815, 121

Dessart, L., Blondin, S., Brown, P. J., et al. 2008, ApJ, 675, 644

Dessart, L., Gutierrez, C. P., Hamuy, M., et al. 2014, MNRAS, 440, 1856

Dessart, L., \& Hillier, D. J. 2005, A\&A, 439, 671

Dessart, L., Hillier, D. J., Waldman, R., \& Livne, E. 2013, MNRAS, 433, 1745

Durbin, W., \& Watson, G. S. 1950, Biometrika, 37, 409

Eastman, R. G., Schmidt, B. P., \& Kirshner, R. 1996, ApJ, 466, 911

Ellis, R. S., Sullivan, M., Nugent, P. E., et al. 2008, ApJ, 674, 51

Faran, T., Poznanski, D., Filippenko, A. V., et al. 2014a, MNRAS, 445, 554

Faran, T., Poznanski, D., Filippenko, A. V., et al. 2014b, MNRAS, 442, 844 Filippenko, A. V. 1997, ARA\&A, 35, 309

Fixsen, D. J., Cheng, E. S., Gales, J. M., et al. 1996, ApJ, 473, 576

Folatelli, G., Morrell, N., Phillips, M. M., et al. 2013, ApJ, 773, 53

Foreman-Mackey, D., Hogg, D. W., Lang, D., \& Goodman, J. 2013, PASP, 125,306

Frieman, J. A., Bassett, B., Becker, A., et al. 2008, AJ, 135, 338

Fukugita, M., Ichikawa, T., Gunn, J. E., et al. 1996, AJ, 111, 1748

Galbany, L., Hamuy, M., Phillips, M. M., et al. 2016, AJ, 151, 33

González-Gaitán, S., Tominaga, N., Molina, J., et al. 2015, MNRAS, 451, 2212

Gunn, J. E., Carr, M., Rockosi, C., et al. 1998, AJ, 116, 3040

Gunn, J. E., Siegmund, W. A., Mannery, E. J., et al. 2006, AJ, 131, 2332

Guy, J., Sullivan, M., Conley, A., et al. 2010, A\&A, 523, A7

Hamuy, M., Folatelli, G., Morrell, N. I., et al. 2006, PASP, 118, 2

Hamuy, M., Phillips, M. M., Suntzeff, N. B., et al. 1996, AJ, 112, 2391

Hamuy, M., Phillips, M. M., Wells, L. A., \& Maza, J. 1993, PASP, 105, 787

Hamuy, M., \& Pinto, P. A. 2002, ApJL, 566, L63

Hamuy, M. A. 2001, PhD thesis, The Univ. Arizona

Holtzman, J. A., Marriner, J., Kessler, R., et al. 2008, AJ, 136, 2306

Howell, D. A. 2011, NatCo, 2, 350

Howell, D. A., Sullivan, M., Perrett, K., et al. 2005, ApJ, 634, 1190

Hsiao, E. Y., Conley, A., Howell, D. A., et al. 2007, ApJ, 663, 1187

Ivezić, Ž., Lupton, R. H., Schlegel, D., et al. 2004, AN, 325, 583

Kasen, D., \& Woosley, S. E. 2009, ApJ, 703, 2205

Kelly, B. C. 2007, ApJ, 665, 1489

Kessler, R., Bassett, B., Belov, P., et al. 2010, PASP, 122, 1415

Kim, A., Goobar, A., \& Perlmutter, S. 1996, PASP, 108, 190

Kirshner, R. P., \& Kwan, J. 1974, ApJ, 193, 27

Kwiatkowski, D., Phillips, P. C. B., Schmidt, P., \& Shin, Y. 1992, Journal of Econometrics, 54, 159

Magnier, E. A., \& Cuillandre, J.-C. 2004, PASP, 116, 449

Minkowski, R. 1941, PASP, 53, 224

Nugent, P., Kim, A., \& Perlmutter, S. 2002, PASP, 114, 803

Nugent, P., Sullivan, M., Ellis, R., et al. 2006, ApJ, 645, 841

Oke, J. B., \& Sandage, A. 1968, ApJ, 154, 21

Olivares, F., Hamuy, M., Pignata, G., et al. 2010, ApJ, 715, 833

Patat, F., Barbon, R., Cappellaro, E., \& Turatto, M. 1994, A\&A, 282, 731

Pejcha, O., \& Prieto, J. L. 2015, ApJ, 799, 215

Perlmutter, S., Aldering, G., Goldhaber, G., et al. 1999, ApJ, 517, 565

Perlmutter, S., Gabi, S., Goldhaber, G., et al. 1997, ApJ, 483, 565

Perrett, K., Balam, D., Sullivan, M., et al. 2010, AJ, 140, 518

Phillips, M. M. 1993, ApJL, 413, L105

Poznanski, D., Butler, N., Filippenko, A. V., et al. 2009, ApJ, 694, 1067

Poznanski, D., Ganeshalingam, M., Silverman, J. M., \& Filippenko, A. V. 2011, MNRAS, 415, L81

Poznanski, D., Nugent, P. E., \& Filippenko, A. V. 2010, ApJ, 721, 956

Regnault, N., Conley, A., Guy, J., et al. 2009, A\&A, 506, 999

Rest, A., Scolnic, D., Foley, R. J., et al. 2014, ApJ, 795, 44

Richardson, D., Jenkins, R. L., III, Wright, J., \& Maddox, L. 2014, AJ, 147,118

Riess, A. G., Filippenko, A. V., Challis, P., et al. 1998, AJ, 116, 1009

Riess, A. G., Press, W. H., \& Kirshner, R. P. 1996, ApJ, 473, 88

Rodríguez, Ó., Clocchiatti, A., \& Hamuy, M. 2014, AJ, 148, 107 
Rubin, D., Knop, R. A., Rykoff, E., et al. 2013, ApJ, 763, 35

Sako, M., Bassett, B., Becker, A. C., et al. 2014, arXiv:1401.3317

Sanders, N. E., Soderberg, A. M., Gezari, S., et al. 2015, ApJ, 799, 208

Schlafly, E. F., \& Finkbeiner, D. P. 2011, ApJ, 737, 103

Schmidt, B. P., Suntzeff, N. B., Phillips, M. M., et al. 1998, ApJ, 507, 46

Scolnic, D., Rest, A., Riess, A., et al. 2014, ApJ, 795, 45

Scovacricchi, D., Nichol, R. C., Bacon, D., Sullivan, M., \& Prajs, S. 2016, MNRAS, 456, 1700

Smartt, S. J., Eldridge, J. J., Crockett, R. M., \& Maund, J. R. 2009, MNRAS, 395, 1409

Smith, J. A., Tucker, D. L., Kent, S., et al. 2002, AJ, 123, 2121

Stephen, M. A. 1974, JASA, 69, 730
Stoughton, C., Lupton, R. H., Bernardi, M., et al. 2002, AJ, 123, 485

Stritzinger, M., Hamuy, M., Suntzeff, N. B., et al. 2002, AJ, 124, 2100

Stritzinger, M. D., Phillips, M. M., Boldt, L. N., et al. 2011, AJ, 142, 156

Sullivan, M., Guy, J., Conley, A., et al. 2011, ApJ, 737, 102

Taddia, F., Moquist, P., Sollerman, J., et al. 2016, A\&A, 587, L7

Takáts, K., \& Vinkó, J. 2012, MNRAS, 419, 2783

Turatto, M., Benetti, S., \& Cappellaro, E. 2003, in From Twilight to Highlight: The Physics of Supernovae, ed. W. Hillebrandt \& B. Leibundgut (Berlin: Springer), 200

Valenti, S., Howell, D. A., Stritzinger, M. D., et al. 2016, MNRAS, 459, 3939 Walker, E. S., Hook, I. M., Sullivan, M., et al. 2011, MNRAS, 410, 1262

York, D. G., Adelman, J., Anderson, J. E., Jr., et al. 2000, AJ, 120, 1579 